\title{
Analyses non invasives in situ des œuvres préhistoriques de la grotte de Font-de-Gaume pour une meilleure connaissance du décor pariétal polychrome et de son organisation
}

Non invasive in situ analyzes of prehistoric rock paintings in the Font-de-

Gaume cave for an improved knowledge of the polychrome parietal decor and of its organization

Ina Reiche, Antoine Trosseau, Katharina Müller, Marine Gay, David Strivay et Jean-Jacques Cleyet-Merle

\section{(2) OpenEdition}

\section{Journals}

Édition électronique

URL : http://journals.openedition.org/paleo/5707

DOI : $10.4000 /$ paleo. 5707

ISSN : 2101-0420

Éditeur

SAMRA

\section{Édition imprimée}

Date de publication : 1 septembre 2020

Pagination : 262-269

ISSN : $1145-3370$

Référence électronique

Ina Reiche, Antoine Trosseau, Katharina Müller, Marine Gay, David Strivay et Jean-Jacques CleyetMerle, « Analyses non invasives in situ des œuvres préhistoriques de la grotte de Font-de-Gaume pour une meilleure connaissance du décor pariétal polychrome et de son organisation », PALEO [En ligne], 30-2 | 2020, mis en ligne le 30 septembre 2020, consulté le 09 décembre 2020. URL : http:// journals.openedition.org/paleo/5707 ; DOI : https://doi.org/10.4000/paleo.5707 


\section{ANALYSES NON INVASIVES \\ IN SITU DES CEUVRES \\ PRÉHISTORIQUES \\ DE LA GROTTE

DE FONT-DE-GAUME

POUR UNE MEILLEURE

CONNAISSANCE

DU DÉCOR PARIÉTAL

POLYCHROME ET

DE SON ORGANISATION

Ina Reichealblc

Antoine Trosseau

Katharina Müllerbc

Marine Gayc

David Strivay $^{\mathrm{d}}$

Jean-Jacques Cleyet-Merlee
Dans cet article est présenté un premier bilan des résultats récents d'analyses chimiques du décor de la grotte de Font-de-Gaume. Ces données ont été obtenues lors de quatre missions de terrain entre 2013 et 2017. Un peu plus de deux cents représentations se répartissent entre un art animalier et des signes (tectiformes), dans des proportions d'environ deux tiers un tiers. L'animal le plus représenté est le bison, d'où le nom la "Caverne des bisons". Les figures ont été réalisées avec différentes techniques. On retrouve la peinture polychrome, le dessin et les gravures. L'état de conservation de la grotte étant le reflet de sa grande fragilité, les parois sont très hétérogènes, ce qui représente un réel défi analytique pour l'analyse chimique des figures dans la mesure où les analyses doivent se limiter à des approches non invasives sans contact. Ainsi, nous avons réalisé environ deux cents analyses par fluorescence $X$ portable pour caractériser les matières colorantes. Elles ont été effectuées dans le but d'obtenir de nouveaux éléments de réponse quant à la composition chimique de la matière colorante utilisée et les techniques de réalisation de ces œuvres du Paléolithique supérieur. Nous présentons ici l'approche utilisée en se focalisant particulièrement sur les traitements des mesures effectuées. Ces premières évaluations des données ont permis d'identifier différentes matières colorantes utilisées dans la grotte de Font-de-Gaume. L'approche développée a ainsi montré son potentiel lorsqu'elle est utilisée sur un plus grand nombre de figures et en intégrant des informations complémentaires. Elle devrait permettre, à terme, d'améliorer la connaissance de ces œuvres et de leur réalisation successive dans le cas de superpositions des figures, ce qui paraît très important en l'absence d'une datation directe des œuvres pariétales de Font-de-Gaume.

a. PSL Université, ENSCP, Institut de recherche de Chimie Paris, UMR 8247 CNRS, PCMTH team - Centre de recherche et de restauration des musées de Franceina.reiche@culture.gouv.fr

b. Rathgen-Forschungslabor, Staatliche Museen zu Berlin, Stiftung Preußischer Kulturbesitz

c. Laboratoire d'Archéologie Moléculaire et Structurale, LAMS, UMR 8220 CNRS

d. Centre européen d'Archéométrie, Université de Liège

e. Musée National de Préhistoire, Les Eyzies

PALE0 30 | t. 2

JUILLET 2020

PAGES 262 À 279

MOTS-CLÉS Art pariétal des grottes ornées, peintures polychromes, oxydes de fer, oxydes de manganèse, fluorescence $X$ portable, analyse non-invasive, traitement quantitatif des données. 
Non invasive in situ analyzes of prehistoric rock

paintings in the Font-de-Gaume cave for an

improved knowledge of the polychrome parietal

decor and of its organization.

In this article we present a first assessment of recent results of chemical analysis of the decor of the Font-de Gaume cave. These data were obtained during four field missions between 2013 and 2017. Slightly more than two hundred representations are divided between animal art and signs (tectiforms), in proportions of about two thirds to one third. Most represented is the bison, hence the name "Bison Cave". The figures were made with different techniques. We find polychrome paintings, drawings and engravings. The state of conservation of the cave is a reflection of its great fragility. The walls are very heterogeneous, which represents a real analytical challenge for the chemical analysis of figures since the analyzes must be limited to non-invasive approaches without contact. Thus, we carried out around two hundred analyzes by portable X-ray fluorescence to characterize the coloring matters. These analyzes were carried out in order to obtain new elements of answer as for the chemical composition of the coloring matter used and the techniques of realization of these works of the Upper Paleolithic. Here we present the approach used, focusing particularly on the processing of the measurements carried out. These first evaluations of the data made it possible to identify different coloring materials used in the Font-de-Gaume cave. The approach developed has thus shown its potential, when applying on a larger number of figures and by integrating additional information. In the long term this enables improving knowledge of these works and their successive realization in the case of superimposed figures which seems very important, in the absence of a direct dating of the parietal works of Font-de-Gaume.

\section{1 | PRÉSENTATION DE LA GROTTE ET DE SON DÉCOR}

Découverte le 12 septembre 1901 par Denis Peyrony, instituteur aux Eyzies-de-Tayac, la grotte de Font-de-Gaume comporte un impressionnant ensemble d'art pariétal. Elle est située à moins d'un kilomètre du village des Eyziesde-Tayac, sur le flanc droit d'une petite vallée qui s'ouvre sur la vallée de la Beune et la surplombe d'une vingtaine de mètres (fig.1). Son réseau karstique bien développé est façonné horizontalement dans le calcaire gréseux de l'étage géologique du Coniacien supérieur (Crétacé supérieur) sur environ 120 mètres, à partir d'une ancienne diaclase, fissure verticale élargie par les eaux, orientée nord-ouest-sud-est. Son creusement, du fait de son altitude relative, daterait au minimum du Pléistocène moyen.

Une première galerie, la galerie d'accès, permet d'atteindre après une étroiture appelée le "Rubicon", située à 65 mètres de l'entrée de la grotte, la galerie principale puis le diverticule final (fig. 1). À ce boyau principal viennent s'ajouter trois diverticules latéraux, les galeries Vidal, Prat et latérale et plusieurs boyaux secondaires généralement inaccessibles. Le tracé de ce réseau est assez régulier. Ses galeries étroites, ponctuées de rétrécissements, conservent une largeur comprise entre 1,50 et 3 mètres. Leur hauteur varie quant à elle, allant de 1,60 à 3,80 mètres dans la galerie d'accès pour atteindre une dizaine de mètres dans la galerie principale.

L'art pariétal de Font-de-Gaume, ou ce qu'il en subsiste, n'est pas réparti uniformément dans la caverne. L'essentiel de cet art se concentre dans la seconde partie de la grotte. Dans la première partie (avant le Rubicon) peu de vestiges du décor ancien sont conservés. Il y a quelques gravures mais peu lisibles, chevaux, tectiformes et bisons associés à des faisceaux de stries, ainsi que des vestiges de dessins rouges ou noirs, chevaux et mammouths. Capitan, Breuil et Peyrony attribuent la rareté du décor à des « conditions de conservations défavorables, soit à l'ouverture de la caverne soit même à une distance plus ou moins considérable de l'entrée » qui auraient détruit les œuvres (Capitan et al. 1910). Frédéric Plassard parle de « conservation différentielle » (Plassard 2005).

Les parois de la seconde partie de la grotte montrent une abondance d'œuvres majeures représentant bisons, mammouths, rennes et deux mains négatives. Les représentations se déroulent en de grandes compositions le long de la galerie principale avec une forte concentration en son début et un rassemblement, à la fin, d'une douzaine de bisons, habillant dans une composition tournante le cabinet des Bisons. À la fin de cette galerie, le diverticule final correspondant à un boyau très étroit et peu accessible rassemble quelques figures d'espèces peu représentées ailleurs dans la grotte. C'est le cas d'un rhinocéros et d'un félin. Enfin, la galerie latérale montre un dispositif pariétal plus pauvre que la galerie principale où le cheval domine et est affectée à son entrée par la formation de concrétions de calcite.
KEY-WORDS Painted cave art, polychrome paints, iron oxides, manganese oxides, portable $X$ ray fluorescence, non-invasive analysis, quantitative data processing 


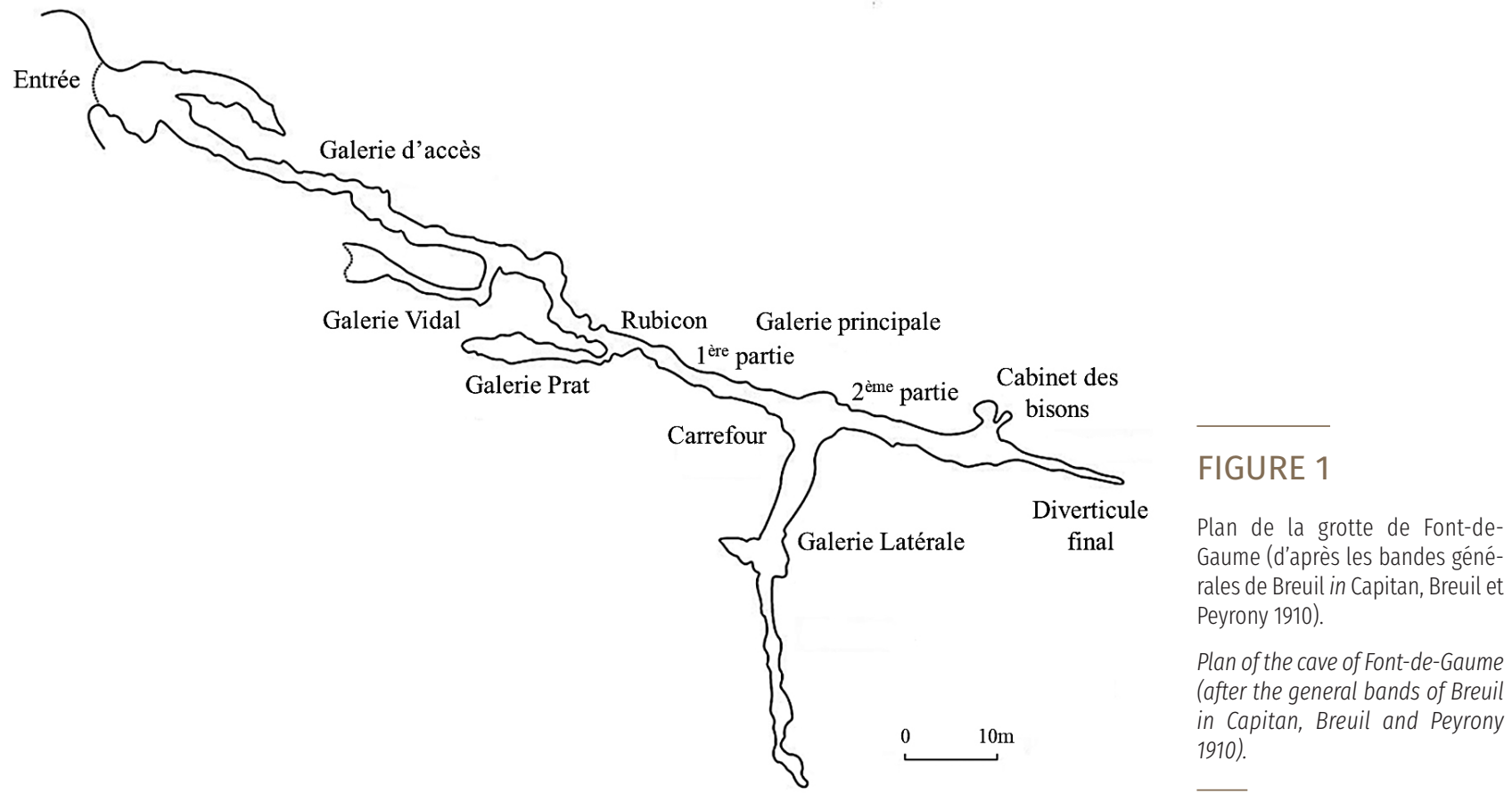

\section{1 | Les techniques}

Différentes techniques artistiques ont été employées à Font-de-Gaume, parfois seules, parfois associées dans une même composition pour un rendu exceptionnel. La peinture s'allie alors au dessin et à la gravure en une technique mixte de réalisation des œuvres. Différentes teintes allant du noir au brun, du rouge au jaune peuvent être observées, les dessins étant principalement réalisés en noir et en rouge. La présence de la couleur jaune est sujette à questionnement. Il n'est pas déterminé si la matière colorante jaune a été apportée à la grotte pour la réalisation du décor ou si les artistes ont profité de la coloration naturelle jaune qui est parfois présente sur les parois pour leur décor.

L'emploi de la gravure, lorsqu'elle est associée aux autres techniques, permet de détourer l'œuvre en accentuant ses contours, de dessiner des détails (œil, cornes, sabots) ou de créer du « blanc ». À cela se rajoute une mise à profit du relief de la paroi par les artistes afin de mettre en volume certaines parties de l'animal représenté.

\section{2 | Les sujets}

L'art pariétal de Font-de-Gaume se compose d'un peu plus de deux cents représentations qui se répartissent entre un art animalier et des signes (tectiformes), dans des proportions d'environ deux tiers un tiers.

La grotte de Font-de-Gaume est connue comme la «Caverne aux bisons ». En effet, le bestiaire représenté à Font-de-Gaume est largement dominé par cet animal avec environ quatre-vingts individus. La facture de grande qualité de ces bovidés contribue à donner à cette grotte son importance. On a ainsi des bisons traités par polychromie, où la peinture et la gravure s'associent aux jeux de relief de la paroi. L'archétype de l'« hyperbison », terme introduit par P. Paillet (Paillet 1993), se retrouve dans beaucoup de ces individus. Il se traduit par un déséquilibre antéropostérieur très prononcé, où l'avant-train est dilaté, le garrot et le décrochement dorso-lombaire amplifiés et le chignon exagéré grâce à une convexité bien marquée. Le bison ci-dessous est l'exemple le plus saisissant de la grotte (fig.2).

Le cheval et le mammouth représentent chacun une quarantaine de sujets, traités de façon plus discrète. Le cheval se distribue dans l'ensemble de l'espace orné, le plus souvent représenté comme «sortant » de la caverne (Cleyet-Merle 2014). Le mammouth est essentiellement gravé et passe donc plus inaperçu. Il se localise surtout dans le début de la galerie principale et sur les parois gauches. Une vingtaine de rennes ou cervidés complètent cet ensemble de représentations, auquel se rajoutent en plus petit nombre des capridés ainsi que, de façon isolée ou par paires, rhinocéros, félin, ours, loup, auroch et forme humaine.

À ce riche bestiaire se rajoutent des mains négatives, quatre au total, ainsi que des signes divers dont vingt-cinq tectiformes qui se déclinent suivant trois morphologies différentes (pentagonal, triangulaire et ramifié). Ces vingtcinq tectiformes, spécifiques à la région, représentent à peu près la moitié des exemplaires connus actuellement (Vialou et Vilhena-Vialou 2014).

\section{3 | Datation de l'art pariétal}

Aucune datation directe de l'art pariétal de Font-deGaume n'a pu être effectuée, en l'absence de tracés au charbon de bois détectés pour le moment. L'estimation de l'âge des figures de la grotte repose donc sur une étude stylistique des panneaux ornés et une étude de la superposition des représentations; l'étude du contexte archéologique n'ayant apporté aucun indice (Cleyet-Merle 2014).

D'après Breuil, la réalisation des œuvres s'accumulerait sur une longue période de temps, depuis l'Aurignacien jusqu'au Magdalénien. La chronologie qu'il propose est basée sur une appréciation de l'évolution au cours du 


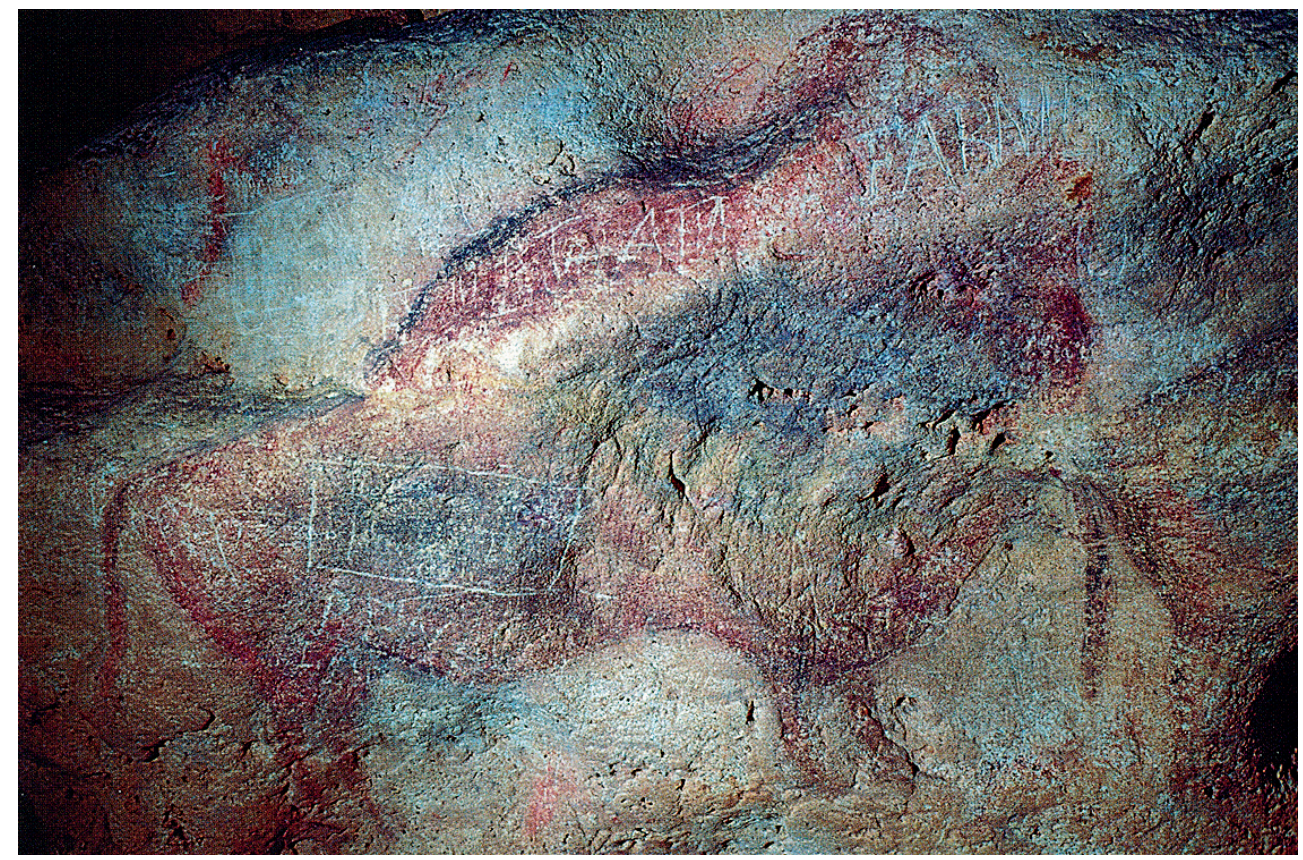

\section{FIGURE 2}

Bison polychrome 43, symbole de l'« hyperbison » (1ère partie de la galerie principale, paroi droite). Source : [Cleyet-Merle 2014]

Polychrome Bison 43, symbol of the " hyperbison " (1st part of the main gallery, right wall). Source: [Cleyet-Merle 2014].

temps de la technique et de la thématique. Ainsi, les figures de la galerie Prat et de la deuxième partie de la Galerie latérale appartiendraient aux phases les plus anciennes (Plassard 2005 ; Capitan et al. 1910 ). A. LeroiGourhan rapporte cette chronologie aux styles III et IV, la recentrant dans le temps. Il attribue ainsi à la partie profonde de la grotte le style III ainsi qu'à un aurochs du cabinet des Bisons et peut-être également aux quelques figures fragmentaires de la galerie Prat; le style IV s'attachant à la partie centrale de l'ornementation (LeroiGourhan et Vertut 1965). J. Clottes évoque, quant à lui, une attribution « dans son ensemble au magdalénien ancien, voir au solutréen final pour une minorité et au magdalénien moyen pour nombre de chercheurs » (Clottes 2008). Enfin, J.-J. Cleyet-Merle parle « d'ultime recours» dans la comparaison du style des œuvres avec d'autres, pariétales ou mobilières, voisines ou lointaines, dont l'âge est connu. Il attribue ainsi le décor pariétal de Font-deGaume à la phase moyenne du magdalénien, vers 15000 ou 14000 ans (Cleyet-Merle 2014).

\section{4 | Historique des recherches physico-chimiques et conservatoires engagées à Font-de-Gaume depuis sa découverte}

Les premières analyses chimiques de la matière picturale de la grotte de Font-de-Gaume ont été réalisées dès 1902 sur des prélèvements peu après la découverte - des poudres noires et rouges, confiés par L. Capitan et H. Breuil à H. Moissan. Les résultats furent publiés dans les comptes rendus hebdomadaires des séances de l'académie des sciences de juin 1902. Selon ces premières analyses, le rouge est composé d'hématite. Le noir est un oxyde de manganèse, contenant aussi du fer. Les matières colorantes sont par ailleurs affectées par des ajouts de silice et de carbonates de calcium, supposés ultérieurs à l'application de matière colorante sur la paroi (Moissan 1902 - p. 1540). Ce furent les seules analyses des matières colorantes de la grotte à notre connaissance avant nos analyses actuelles.
D'autres analyses ont été initiées depuis, touchant à la conservation de cette cavité. Des études menées par le pôle grottes ornées du Laboratoire de Recherche des Monuments Historiques (LRMH) se sont attachées dans un premier temps à évaluer la nature d'altérations d'origine biologique pour les traiter. Depuis les années 1990, une politique de prévention est préférée et appliquée pour maintenir l'équilibre naturel de la grotte. Un suivi climatique et un contrôle biologique régulier, sans traitement biocide, ont ainsi été instaurés pour assurer une bonne gestion de ce milieu karstique.

Récemment, une recherche portant sur le développement de vermiculations affectant les parois ornées de la grotte, a été menée par le Centre National de Préhistoire (CNP). Celles-ci mobilisent en effet des matériaux en surface des parois (matériaux naturels, pigments) et pouvant potentiellement menacer la conservation des œuvres pariétales (Konik et al. 2014).

\section{I PROBLÉMATIQUE DE LA RECHERCHE ACTUELLE}

Beaucoup de travaux visent à identifier les matières colorantes employées durant le Paléolithique, notamment lorsqu'elles sont appliquées sur des parois (Huntley et al. 2015 ; Chanteraud et al. 2019 ; Mauran et al. 2019 ; Gay et al. 2020). En l'absence d'une datation directe des figures, ces recherches ont pour but d'apporter de nouveaux éléments de réponse quant à la séquence de réalisations des figures préhistoriques, les techniques utilisées et leur état de conservation afin d'améliorer la compréhension du décor pariétal de la caverne. Les contraintes patrimoniales visant la préservation de l'intégrité des représentations rupestres nous ont conduit à élaborer une stratégie de caractérisation non-invasive in situ de la composition chimique des matières colorantes des peintures et dessins de Font-de-Gaume. Cette démarche a été engagée depuis 2013 en employant essentiellement des analyses par fluorescence X portable (FX). D'une façon générale, il est préférable et nécessaire de coupler cette 
approche à d'autres observations fines et complémentaires de la paroi. Ce couplage est prévu pour la suite de notre programme de recherche. Ce premier travail à la grotte de Font-de-Gaume avait donc pour but de mieux évaluer le potentiel informatif des analyses chimiques in situ dans ce contexte précis et de réfléchir concrètement sur une contextualisation des données chimiques en vue de disposer de nouveaux éléments de réponse quant à la réalisation et à la signification des figures préhistoriques. Conscients des limites de cette approche analytique nos interprétations des données présentées se limitent actuellement à des tests de faisabilité et d'optimisation des traitements de données de la FX portable afin de disposer d'un jeu de données fiables qui, en tentant compte du contexte et d'autres informations, nous permettra à terme de mieux comprendre la constitution, l'état de préservation et la signification du décor pariétal de la caverne tel que cela a pu être réalisé pour la grotte de Rouffignac (Gay et al. 2020).

Différentes hypothèses de travail devront être discutées à ces fins notamment s'il est possible d'identifier d'une façon semi-quantitative une signature chimique de la matière colorante des figures préhistoriques in situ, si les caractéristiques de la matière colorante employée peuvent donner des indications sur la séquence de création des figures ou permettent l'attribution des figures à différentes mains, etc. Il est certain qu'il est nécessaire de croiser ces données avec d'autres informations telles que les styles, les superpositions de figures, l'état de la paroi avant de conclure sur les implications chronoculturelles de telles analyses.

Au moins, nous pouvons postuler que la comparaison des signatures physico-chimiques d'une image à l'autre devrait permettre de différencier ou de rassembler certaines figures et de vérifier si le même type d'animal ou la figure du même style a été réalisé avec la même matière colorante.

Ces recherches sur l'identification de la matière picturale accompagnent les travaux portant sur la taphonomie des parois. Ce volet est réalisé en étroite collaboration avec le CNP (Centre National de Préhistoire) et le LRMH (Laboratoire de recherche des monuments historiques).

\section{3 | MÉTHODOLOGIE MISE EN CEUVRE}

\section{1 | Approche non invasive}

Dans le travail présenté ici nous considérons la composition chimique des couches picturales et comment il est possible de l'analyser. Vu la fragilité du site et des parois ornées, les analyses ont été limitées à des mesures non-invasives en utilisant un équipement portable. Ces équipements ont déjà fait leurs preuves dans la grotte de Rouffignac et ont permis de caractériser finement le décor noir de cette grotte (Beck et al. 2012, 2014 ; Lalhil et al. 2012 ; Gay et al. 2015 ; Gay et al. 2020).

À ce jour, quatre missions ont été effectuées à la grotte de Font-de-Gaume pour conduire les analyses chimiques non invasives de son art pariétal (tabl.1).

\section{2 | Méthodes employées}

Les mesures en spectrométrie de fluorescence $X$ (FX) permettent d'identifier la composition en éléments chimiques du matériau analysé. Outre leur caractère non invasif, ces mesures ont une sensibilité suffisante pour permettre, selon les cas de figure, une différenciation entre les matières colorantes. Grâce à ces analyses, il est donc envisageable de distinguer les figures analysées selon la composition chimique de leur matière colorante et ainsi de faire des regroupements ou distinctions non visibles à l'œil nu.

Quelques mesures ont été réalisées à l'aide de la FX 2D, notamment sur le bison $18 \mathrm{~b}$ et le bison 48 , sur une zone de $5 \times 5 \mathrm{~cm}^{2}$. Ces mesures permettent d'obtenir des cartographies chimiques et fournissent ainsi des informations sur la répartition des éléments chimiques dans les tracés colorés et la paroi. L'appareil de FX 2D a été mis à disposition par le Centre européen d'Archéométrie de Liège (Strivay et al. 2016).

La spectrométrie Raman permet de détecter les traces de carbone et d'identifier différentes phases de la matière colorante. Ainsi, il est possible de différencier les différentes phases à base d'oxyde de manganèse et de fer pouvant être présentes dans les tracés noirs, rouges, bruns ou jaunes. L'analyse par spectrométrie Raman a été réalisée avec l'appareil mis à disposition par le Centre européen d'Archéométrie de Liège.

\begin{tabular}{|l|l|l|}
\hline Mission & Date & Technique utilisée \\
\hline 1 & 12-14 Novembre 2013 & $\begin{array}{l}\text { Spectrométrie de fluorescence } X^{a} \\
\text { Spectrométrie de fluorescence } X^{a} D^{b} \\
\text { Spectrométrie Raman }\end{array}$ \\
\hline 2 & 12-14 Novembre 2014 & Spectrométrie de fluorescence $X^{\mathrm{a}}$ \\
\hline 3 & 08-10 Février 2016 & Spectrométrie de fluorescence $X^{\mathrm{a}}$ \\
\hline 4 & 09-11 Mars 2017 & Spectrométrie de fluorescence $X^{c}$ \\
\hline
\end{tabular}

a : Laboratoire d'Archéologie Moléculaire et Structurale, UMR 8220 CNRS Université Pierre et Marie Curie Paris VI

b : Centre Européen d'Archéométrie, Université de Liège

c : Staatliche Museen zu Berlin, Stiftung Preußischer Kulturbesitz

\section{TABLEAU 1}

Dates et techniques portables in situ employées lors des missions de terrain.

Dates and in situ portable techniques used during field missions. 


\subsection{Conditions analytiques}

\section{de la fluorescence $\mathrm{X}$ portable}

Les mesures de FX portable ont été effectuées grâce à deux appareils différents. Le premier appareil mis à disposition par le LAMS en 2013, 2014 et 2016 correspond à un appareil portable non commercial construit sur mesure (Gay et al. 2020). Ce spectromètre était bien adapté aux conditions de travail particulières en grotte, en raison de sa grande flexibilité, de son poids minimal et de sa facilité de manipulation. Cela était d'autant plus important que les peintures et dessins n'étaient pas facilement accessibles sur les parois de la grotte. Le spectromètre principal est doté d'un tube à rayons X MOXTEK de $40 \mathrm{kV}$ avec une anode en palladium et d'une taille de faisceau d'environ $1 \mathrm{~mm}^{2}$ sur l'échantillon obtenu à l'aide d'un collimateur. Un détecteur SDD de $7 \mathrm{~mm}^{2}$ avec une résolution en énergie de 140 eV (FWHM à 5,9 keV) a été utilisé pour collecter les signaux de FX. Le tube à rayons $X$ et le détecteur sont fixés à $45^{\circ} / 90^{\circ}$ sur un système de positionnement permettant des mouvements micrométriques. La tête du détecteur est à une distance de $5 \mathrm{~mm}$ de la surface analysée. On repère celle-ci à l'aide d'un laser basse puissance et des caméras permettent de photographier sa position. Les conditions de mesure étaient 30 kV, 300 HA et 300 s / mesure. Trois spectres représentatifs de FX mesurés avec l'appareil du LAMS sur différents points d'analyse du bison sont représentés dans la figure 3. Le spectre de FX présente des pics de différentes énergies, caractéristiques des éléments chimiques majeurs et mineurs présents dans le tracé et la paroi. Les éléments traces sont difficilement détectés car leur signal est généralement trop peu intense. Les trois spectres obtenus sur une même figure illustrent l'hétérogénéité des réponses malgré la similitude de la composition chimique attendue. Ceci est lié à l'hétérogénéité locale de la paroi et à la géométrie de l'analyse, variable en fonction du support.

Le deuxième appareil portable utilisé lors de la mission en 2017 est un instrument commercial Elio/XGlab ${ }^{\circledR}$. Cet appareil standardisé contient un tube à rayons $\mathrm{X}$ de $50 \mathrm{kV}$ avec une anode en rhodium pouvant délivrer une puissance de $4 \mathrm{~W}$. Le détecteur est un détecteur SDD de $17 \mathrm{~mm}^{2}$ dont la résolution en énergie est de $140 \mathrm{eV}$. Il est également équipé d'un collimateur capable de focaliser le faisceau sur un spot de $1 \mathrm{~mm} 2$, de deux lasers, d'un système de positionnement et d'un trépied. De plus, il bénéficie d'une caméra. La distance de travail est de l'ordre de $5 \mathrm{~mm}$. Les conditions analytiques employées étaient 50 kV et 300 s / mesure. L'appareil Elio montre une meilleure sensibilité d'analyse que celui du LAMS, notamment en ce qui concerne les éléments traces (fig.4). Pour chaque figure préhistorique, entre trois et cinq analyses ponctuelles ont été réalisées, combinées à quelques points d'analyse sur la paroi à proximité des tracés afin de vérifier l'homogénéité de la composition chimique par figure et d'obtenir des données représentatives pour chaque figure analysée. Les analyses des échantillons standards comme le Brill A (Corning glass) de composition chimique connue ont été effectuées en début et de fin de journée d'analyse en grotte pour s'assurer du bon fonctionnement du spectromètre et de

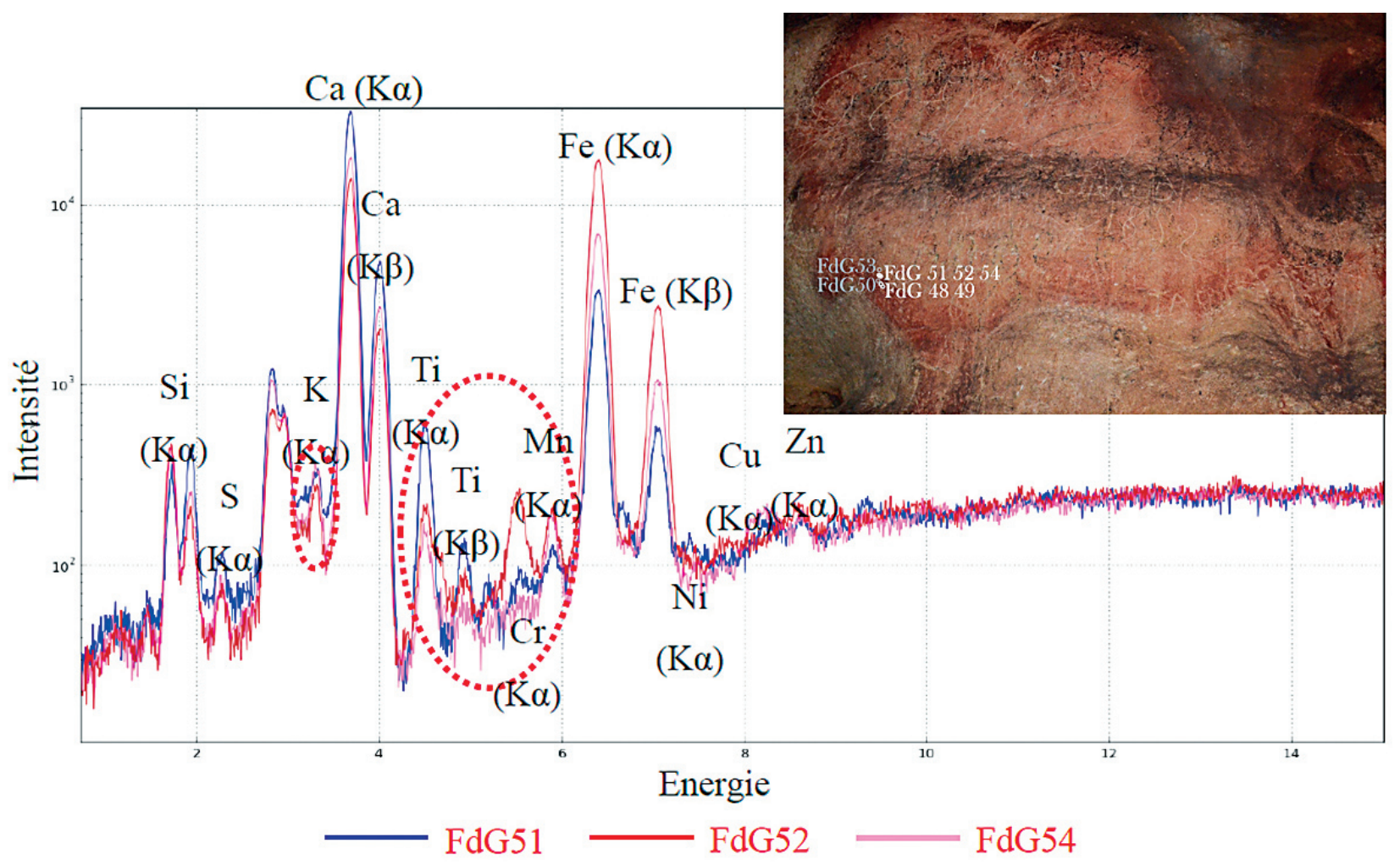

\section{- FIGURE 3 -}

Spectres de fluorescence X (Appareil LAMS) des tracés rouges du bison 4 (points d'analyse FdG51, 52 et 54, peu distants), campagne 2014, O M. Gay, LAMS.
Portable X-ray fluorescence spectrum (LAMS) of the red bison 4 (analysis points: FdG51, 52 and 54, near distant), 2014 campaign, $\odot$ M. Gay, LAMS. 


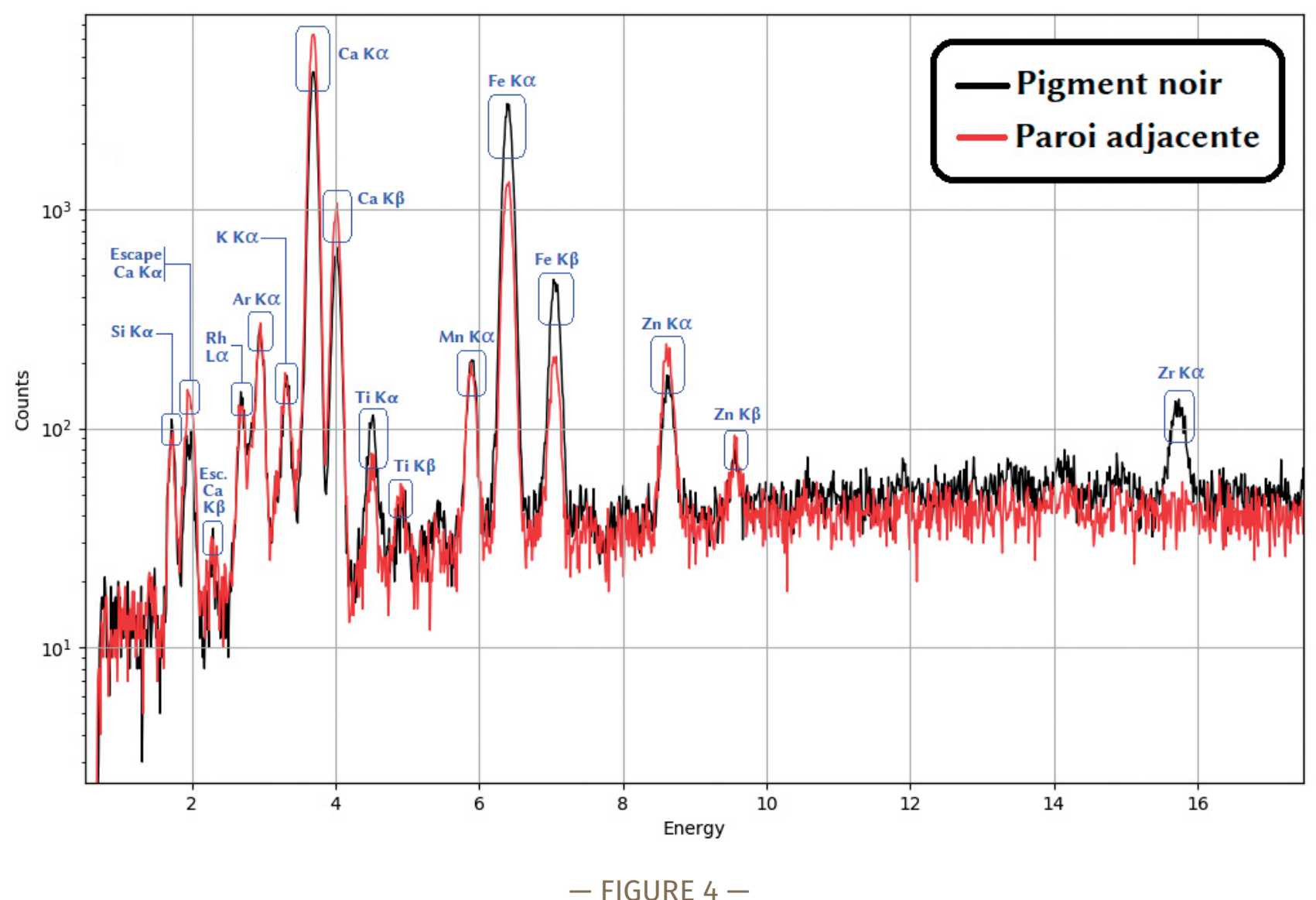

Spectres de fluorescence X (Appareil Elio, XGlab ${ }^{\circledR}$ ) d'un tracé noir de la frise des mammouths et de la paroi correspondante, campagne 2017, (c) A. Trosseau, IRCP/C2RMF.
X-ray fluorescence spectra (Elio Apparatus, XGlab ${ }^{\circledR}$ ) of a black outline of the mammoth frieze and the corresponding wall, 2017 campaign, ( $)$ A. Trosseau, IRCP/C2RMF. la fiabilité des conditions analytiques utilisées. Dans le cas où une différence non négligeable serait constatée entre leurs spectres, une correction simple peut être calculée mathématiquement puis appliquée à tous les points expérimentaux. Pour le passage d'un appareil à l'autre, la correction est plus complexe et s'appuie sur notre connaissance des caractéristiques des appareils.

À l'aide d'un traitement informatique des spectres FX par PyMca, les proportions des éléments détectés sous forme d'oxydes simples peuvent être déterminées et servent à définir la signature chimique caractéristique de la figure. La principale difficulté de l'évaluation des analyses réalisées par FX in situ est la capacité à différencier, pour un point d'analyse sur le tracé, le signal des pigments de celui de la paroi. En effet, les rayons $X$ pénètrent la paroi au-delà de la surface et le signal de FX peut donc détecter des couches sous la surface (DE SANOIT et al. 2005). Cela implique que lorsqu'un tracé est analysé, le spectre contient des informations chimiques sur les matières colorantes et la paroi environnante que l'on ne peut pas aisément séparer.

Les matières colorantes sont présentes en faibles concentrations malgré leur couleur intense et le tracé ne correspond pas à une couche pigmentaire homogène et continue sur la surface de la paroi. La détermination de la composition chimique des pigments représente donc un défi, d'autant plus que les matières colorantes peuvent contenir en grande partie les mêmes éléments chimiques que la paroi, notamment le fer. Chaque spectre mesuré est donc analysé avec précaution.

\section{Corpus étudié jusqu'à présent par fluorescence X portable}

Lors des précédentes missions, un total de 204 points a été analysé par FX portable, dont 70 sur la paroi, 70 sur des tracés rouges, 43 sur des tracés noirs et le reste sur des tracés jaunes ou polychromes. Ces mesures correspondent à un ensemble de 19 figures analysées reparties dans la galerie principale entre le Rubicon et le diverticule final (tabl.2, fig. 5). Ce corpus est donc formé de 12 bisons, 3 tectiformes, 2 mammouths, 1 cheval et 1 main négative. Chaque figure est analysée en plusieurs points, entre 3 et 15 points avec systématiquement plusieurs mesures de la paroi adjacente. Ainsi, un dixième des, environ, 220 figures recensées ont déjà été caractérisées. 


\begin{tabular}{|c|c|c|c|c|}
\hline Année & 2013 & 2014 & 2016 & 2017 \\
\hline \multirow[t]{7}{*}{$\begin{array}{l}\text { Figures } \\
\text { et points } \\
\text { analysés } \\
\text { par } \\
\text { couleur }\end{array}$} & $\begin{array}{l}\text { Main négative } \\
\text { noire } \\
(3+\text { paroi })\end{array}$ & $\begin{array}{l}\text { Bison rouge } 5 \\
(5+\text { paroi })\end{array}$ & $\begin{array}{l}\text { Bison rouge } \\
9 \\
(5+\text { paroi })\end{array}$ & $\begin{array}{l}\text { Bison } 45 \\
\text { (rouge : } 9+\text { paroi, } \\
\text { jaune/brun : } 6+ \\
\text { paroi, } \\
\text { noir : } 7+\text { paroi) }\end{array}$ \\
\hline & $\begin{array}{l}\text { Tectiforme } \\
\text { rouge I } \\
(4+\text { paroi })\end{array}$ & $\begin{array}{l}\text { Petit Cheval noir } \\
(4+\text { paroi })\end{array}$ & $\begin{array}{l}\text { Bison noir } \\
10 \\
(6+\text { paroi })\end{array}$ & $\begin{array}{l}\text { Bison rouge } 12 \\
(5+\text { paroi })\end{array}$ \\
\hline & $\begin{array}{l}\text { Bison } 48 \\
\text { (rouge : } 2+ \\
\text { paroi, noir : } 4+ \\
\text { paroi) }\end{array}$ & $\begin{array}{l}\text { Bison rouge } 4 \\
(5+\text { paroi })\end{array}$ & $\begin{array}{l}\text { Bison } 27 \\
\text { (rouge : } 3 \text {, } \\
\text { noir : } 3+ \\
\text { paroi) }\end{array}$ & $\begin{array}{l}\text { Frise de } \\
\text { Mammouths noire } \\
(3+\text { paroi })\end{array}$ \\
\hline & $\begin{array}{l}\text { Bison 18b } \\
\text { (brûnatre : } 11+ \\
\text { paroi, noir : } 2+ \\
\text { paroi) }\end{array}$ & $\begin{array}{l}\text { Bison } 39 \\
\text { (rouge : } 5+\text { paroi, } \\
\text { noir : } 5+\text { paroi) }\end{array}$ & & $\begin{array}{l}\text { Bison rouge } 40 \\
(4+\text { paroi })\end{array}$ \\
\hline & $\begin{array}{l}\text { Bison } 43 \\
\text { (rouge : } 4+ \\
\text { paroi, noir : } 6+ \\
\text { paroi) }\end{array}$ & $\begin{array}{l}\text { Bison } 38 \\
\text { (rouge : } 2+\text { paroi, } \\
\text { noir : } 3+\text { paroi) }\end{array}$ & & \\
\hline & & $\begin{array}{l}\text { Tectiforme rouge } \\
\text { III } \\
(6+\text { paroi })\end{array}$ & & \\
\hline & & $\begin{array}{l}\text { Tectiforme rouge } \\
\text { II }(10+\text { paroi })\end{array}$ & & \\
\hline
\end{tabular}

\section{TABLEAU 2}

Figures de la grotte de Font-de-Gaume analysées par fluorescence X portable in situ lors des missions de terrain entre 2013 et 2017.

Figures of the Font-de-Gaume cave analyzed in-situ by portable X-ray fluorescence during field missions between 2013 and 2017.

Grotte de Font-de-Gaume

Répartition des figures

(d'après les bandes générales de Breuil in Capitan, Breuil et peyrony, 1910)

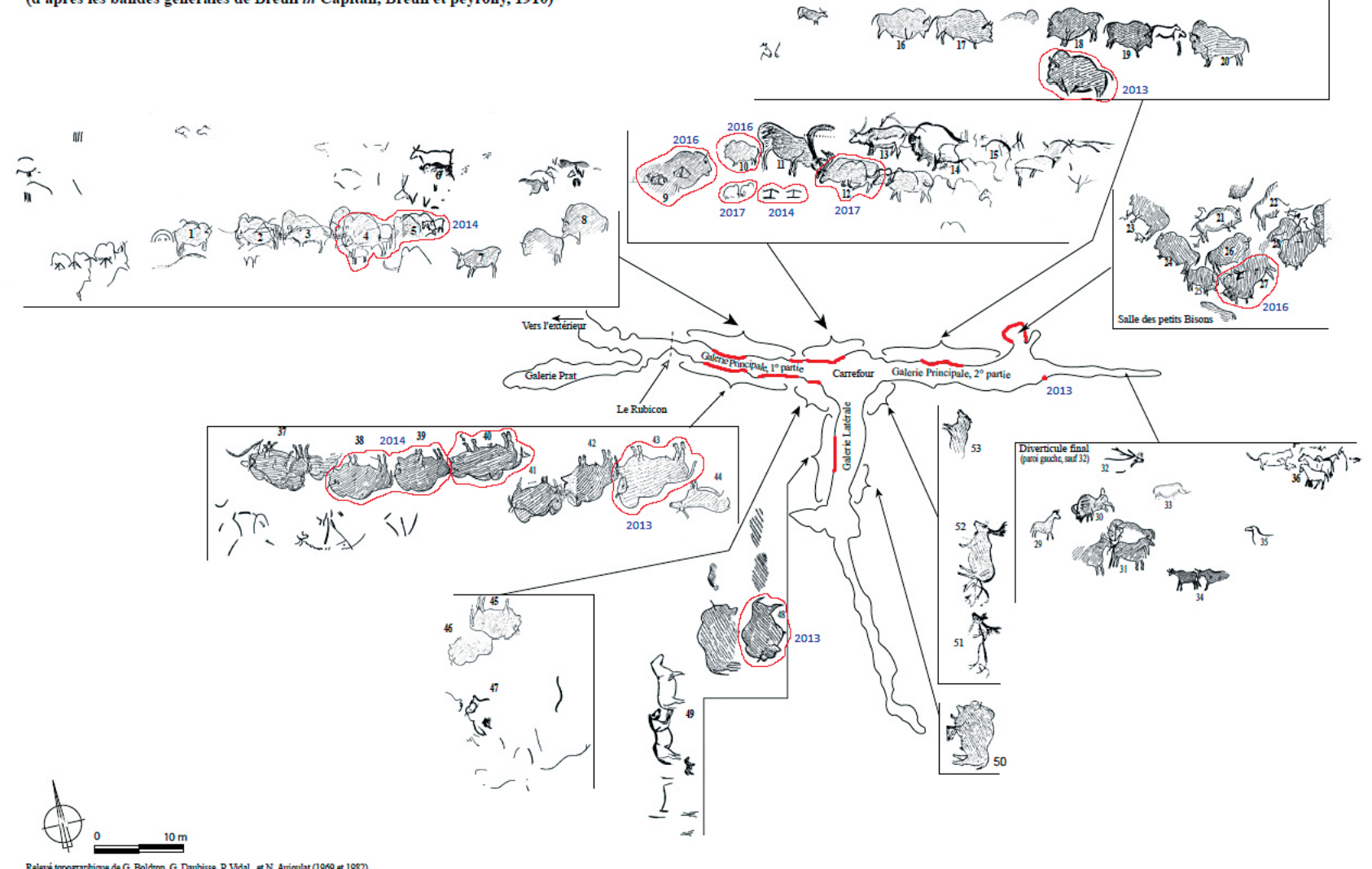

- FIGURE $5-$

Répartition des 19 figures analysées dans la grotte de Font-de-Gaume entre 2013

Distribution of the 19 figures analyzed in the cave of Font-de-Gaume between 2013 et 2017 . and 2017. 


\section{4 | RÉSULTATS PRÉLIMINAIRES}

À ce jour, seule une partie des données issues des missions a pu être traitée. Ces résultats préliminaires proviennent de la thèse de Marine Gay (2015) et de la thèse en cours d'Antoine Trosseau.

Les analyses Raman n'ont pas pour le moment permis d'identifier différentes phases d'oxydes de fer et de manganèse ni de traces de carbone dans les tracés des figures de la grotte. Les analyses en FX 2D n'ont pas encore pu être traitées.

Ainsi, les données présentées dans ce bilan proviennent exclusivement des analyses ponctuelles de la FX portable. Comme expliqué plus haut, le traitement des données de FX portable in situ n'est pas évident et différents modèles de traitement des données doivent être développés en fonction du type de matières colorantes présentes.

\section{1 | Analyse qualitative}

Les tracés rouges, noirs et jaunes ainsi que la paroi contiennent différents éléments chimiques, bien que l'on retrouve sensiblement les mêmes éléments chimiques mais avec différentes proportions dans la paroi et dans les tracés colorés. Le tableau 3 résume les éléments chimiques détectés dans la paroi et les matières colorantes. Comme attendu, la paroi contient majoritairement du calcium, le noir du manganèse (oxyhydroxydes de manganèse) et le rouge et le jaune du fer (oxyde et oxy-hydroxyde de fer).

\subsection{1 | Caractérisation du substrat}

Une analyse systématique de la paroi a accompagné les analyses de la matière picturale. Il en est ressorti une paroi composée majoritairement de calcium. Les autres éléments chimiques présents sont ceux qui ont été également identifiés dans les mélanges picturaux rouge et noir : le silicium, le soufre, le potassium, le titane, le manganèse, le fer, le cuivre et le zinc. Ces éléments sont présents en proportions variables sur les spectres, comme en témoignent les quatre spectres de la figure 6 qui nous montrent un substrat très hétérogène de par sa composition chimique, sur une zone somme toute très restreinte.

\subsection{2 | Caractérisation des mélanges pigmentaires noirs}

Dans le cas du noir, les analyses de FX effectuées ont permis d'identifier le manganèse comme étant le constituant principal du pigment, associé majoritairement à du fer et à du baryum dans certains cas. Ces analyses mettent également en évidence la présence d'autres éléments chimiques, en faibles teneurs pour le silicium, le soufre, le potassium et le titane, et sous forme de traces pour le nickel, le cuivre et le zinc. Nous considérerons par la suite que le calcium et le silicium proviennent essentiellement de la paroi sous-jacente en raison de la nature du substrat. La grotte est en effet creusée dans un calcaire bioclastique gréseux du Coniacien. Les autres éléments détectés peuvent être soit associés à la matière colorante ou à des produits d'altération. Par exemple la présence de soufre peut être liée à des processus de biodégradation (fig. 8).

Les tracés noirs ont pu être assez bien distingués qualitativement en au moins deux groupes. Ainsi, les bisons 38 et 43 par exemple ont été réalisés avec une matière colorante à base de manganèse associé à du fer et du baryum, tandis que le bison 39 contient du manganèse simple. Les oxydes de manganèse contenant des teneurs importantes en baryum sont connus, comme la hollandite $(\mathrm{Ba})_{1-2} \mathrm{Mn} 8 \mathrm{O}_{10}, \mathrm{xH}_{2} \mathrm{O}$ ) et la romanéchite $\left(\mathrm{Ba}_{2} \mathrm{Mn}_{5} \mathrm{O}_{10}, \mathrm{XH}_{2} \mathrm{O}\right)$. Ces phases ont pu être identifiées dans les matières colorantes issues de la grotte de Lascaux (Chalmin et al. 2003) et à la grotte de Rouffignac (Beck et al. 2012 ; Lahlil et al. 2012 ; Beck et al. 2014).

\subsection{3 | Caractérisation des mélanges pigmentaires rouges et jaunes}

L'élément fer est le principal constituant de cette matière picturale rouge et jaune. D'autres éléments chimiques présents en plus faibles teneurs ont été détectés, tels que le silicium, le soufre, le potassium, le titane et le manganèse. Des éléments traces sont également présents,

\begin{tabular}{|c|c|c|c|c|}
\hline Matière analysée & Paroi & Tracé rouge & Tracé noir & Tracé jaune \\
\hline Éléments majeurs & Calcium & Fer & $\begin{array}{l}\text { Manganèse } \\
\text { Fer } \\
\text { Baryum }\end{array}$ & Fer \\
\hline Éléments mineurs & \begin{tabular}{|l} 
Silicium \\
Soufre \\
Potassium \\
Titane \\
Manganèse
\end{tabular} & $\begin{array}{l}\text { Silicium } \\
\text { Soufre } \\
\text { Potassium } \\
\text { Titane } \\
\text { Manganèse }\end{array}$ & \begin{tabular}{|l|} 
Silicium \\
Soufre \\
Potassium \\
Titane
\end{tabular} & $\begin{array}{l}\text { Silicium } \\
\text { Soufre } \\
\text { Potassium } \\
\text { Titane } \\
\text { Manganèse }\end{array}$ \\
\hline Traces & $\begin{array}{l}\text { Fer } \\
\text { Cuivre } \\
\text { Zinc }\end{array}$ & $\begin{array}{l}\text { Chrome } \\
\text { Cuivre } \\
\text { Zinc }\end{array}$ & $\begin{array}{l}\text { Cuivre } \\
\text { Zinc } \\
\text { Zirconium }\end{array}$ & $\begin{array}{l}\text { Chrome } \\
\text { Cuivre } \\
\text { Zinc }\end{array}$ \\
\hline Homogénéité & $\begin{array}{l}\text { Très } \\
\text { hétérogène }\end{array}$ & $\begin{array}{l}\text { Peu } \\
\text { homogène }\end{array}$ & $\begin{array}{l}\text { Deux groupes } \\
\text { homogènes }\end{array}$ & Peu homogène \\
\hline
\end{tabular}

\section{TABLEAU 3}

Éléments chimiques détectés dans les figures de Font-deGaume (en gras, élément principal.

Chemical elements detected in the figures of Font-de-Gaume (in bold, main element). 


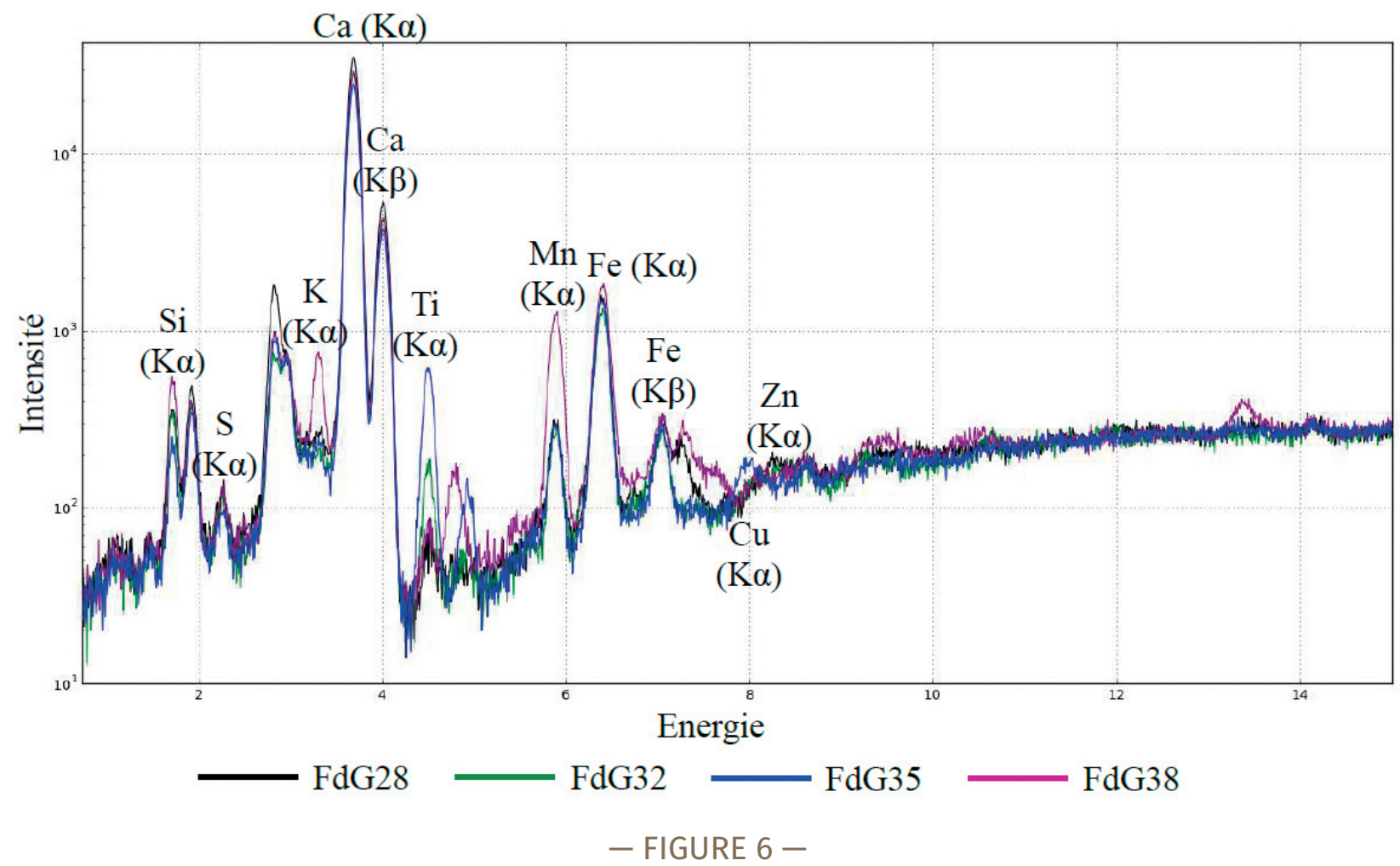

Spectres de FX obtenus sur une zone restreinte située au niveau des pattes avant du bison 39 qui témoignent d'une forte hétérogénéité de la paroi (cf. figure 7), (c) M. Gay, LAMS.

$X$-ray fluorescence spectra obtained on a restricted zone located at the level of the front legs of the bison 39, which testify to a strong heterogeneity of the wall (see figure 7), @ M. Gay, LAMS.

le chrome, le nickel, le cuivre et le zinc. La présence du calcium est uniquement attribuée à la contribution de la paroi sous-jacente, pour faciliter quelque peu le traitement informatique des données (fig.3).

Différentes teintes rouges ont été observées parmi les figures polychromes de la grotte. L'ensemble des spectres FX recueillis témoigne de l'utilisation de matières colorantes à base de fer pour la réalisation de ces figures rouges (probablement sous forme d'oxyde) sans qu'il soit aisé de discriminer précisément la composition chimique de chaque teinte rouge. Il est notamment difficile de trancher entre l'emploi d'un certain oxyde ou d'un autre, du fait de la présence naturelle d'aplats d'argile en beaucoup d'endroits de la paroi et très probablement aussi sous le tracé.

\section{2 | Analyse semi-quantitative}

Différents traitements informatiques ont été appliqués et ont permis d'estimer plus ou moins précisément la quantité d'éléments détectés. Il est nécessaire de distinguer différents cas de figure en fonction du type de décor (dessin ou peinture), de l'homogénéité de la paroi et du type de matière colorante. En règle générale, l'analyse semi-quantitative des matières colorantes des tracés noirs est plus simple car les principaux éléments de la matière colorante se distinguent nettement de ceux contenus dans la paroi. Afin d'extraire des informations semi-quantitatives, le manganèse, le baryum et le fer pouvant être concentrés dans les matières colorantes

noires sont exprimés sous forme d'oxydes. Le traitement sous PyMca implique en effet l'utilisation d'oxydes et non d'éléments chimiques seuls.

Pour le cas le plus simple, des dessins noirs sur une paroi relativement homogène, il est possible d'utiliser une approximation relativement simple pour déterminer d'une façon semi-quantitative la composition des matières colorantes (Beck et al. 2012 ; BECK et al. 2014). Les concentrations des trois éléments $\mathrm{Mn}$, Ba et Fe sont extraites à l'aide du logiciel PyMca puis bouclées sous forme d'oxydes à $100 \%$ car les spectres des tracés noirs sont suffisamment différents de ceux de la paroi pour faire cette approximation. Cette approche semi-quantitative a été déjà adoptée par BECK et al. 2012, 2014 ainsi que par GAY et al. 2020 pour déterminer la composition chimique des dessins noirs de la grotte de Rouffignac, Dordogne. À la grotte de Font-de-Gaume, cette approche semble difficilement applicable car la paroi est très hétérogène, les tracés noirs sont parfois superposés à de la peinture et une partie de la peinture du décor est réalisée avec du noir. De plus, l'état de conservation des œuvres de la grotte de Font-de-Gaume, "affadies" par le temps pour reprendre le terme employé par J.-J. Cleyet-Merle (CleyetMerle 2014), où la couleur a été lessivée à certains endroits, nécessite le développement d'une stratégie de traitement des données plus élaborée (GAY et al. 2016).

\subsection{1 | Traitement des données du noir}

Les rayons $X$ ont la propriété de pénétrer en profondeur dans la matière, ainsi l'analyse d'un tracé en surface est faussée par la contribution du signal du substrat. Cet effet 
est généralement annulé en soustrayant un spectre du substrat à celui du point d'analyse (De Sanoit et al. 2005). Or à Font-de-Gaume, le fait que la paroi soit très hétérogène d'un point à l'autre empêche de comparer les points d'analyses (fig. 7 et 8). Heureusement, la quantité d'oxyde de manganèse contenue dans la paroi est négligeable face à celle des pigments.

Cela a été vérifié par le calcul de la distribution de concentration de manganèse dans le volume total analysé (fig.9). On voit en effet que les points d'analyse des zones colorées contiennent bien plus de manganèse que ceux de la paroi. Toutefois, près de la moitié des points des tracés noirs affichent une part de manganèse inférieure à $5 \%$ de la masse analysée.

De ce fait, pour les tracés noirs suffisamment préservés, il est acceptable de considérer que tout le manganèse provient de la matière colorante. Cela permet de normaliser les concentrations élémentaires obtenues avec le logiciel PyMca par la concentration de manganèse, et ainsi de comparer les teneurs des différents éléments dans la matière colorante. Cette comparaison n'est en revanche valable que pour les éléments dont la quantité dans le tracé coloré est très supérieure à celle du substrat. Parmi les éléments détectés, seul l'oxyde de baryum remplit cette condition. Il se trouve qu'il correspond en effet à certaines phases minérales qui associent oxydes de manganèse et de baryum : la hollandite $\left((\mathrm{Ba})_{1-2} \mathrm{Mn}_{8} \mathrm{O}_{10}, \mathrm{xH}_{2} \mathrm{O}\right)$ et la romanéchite $\left(\mathrm{Ba}_{2} \mathrm{Mn}_{5} \mathrm{O}_{10}, \mathrm{XH}_{2} \mathrm{O}\right)$.

Il a donc été possible de distinguer trois groupes de matières colorantes noires utilisées dans les figures analysées (fig.9). Le premier groupe est formé d'un minéral contenant du manganèse et du baryum, en particulier avec une stœchiométrie quasi-constante de deux atomes de baryum pour trois de manganèse,ce qui laisse penser qu'il s'agirait de la romanéchite. Le deuxième utilise un oxyde de manganèse pur, probablement de la pyrolusite $\left(\mathrm{MnO}_{2}\right)$, la todorokite $\left((\mathrm{Ca}, \mathrm{Na}, \mathrm{K})\left(\mathrm{Mg}, \mathrm{Mn}^{2}+\right) \mathrm{Mn}_{5} \mathrm{O}_{12}, 5 \mathrm{H}_{2} \mathrm{O}\right)$ ou la manganite $(\mathrm{MnO}(\mathrm{OH}))$ qui sont aussi possibles (Chalmin et al. 2003 ; SALOMON et al. 2014) étant bien plus rares dans ce contexte. Un troisième groupe contient une teneur intermédiaire et variable en baryum, il s'agit donc soit d'un mélange des deux premiers groupes, soit d'une matière première contenant déjà plusieurs types d'oxydes de manganèse comme par exemple un mélange naturel de pyrolusite et de romanéchite.

Le bison 45 contient de la matière du groupe 1, mais aussi du groupe 3, ce qui incite à conclure sur l'utilisation de deux matières colorantes mélangées pour le groupe 3. La main négative près du bison 39 appartient également au groupe 3. La matière colorante noire des bisons 39 et 18b, et du petit cheval est issue du même groupe 2 (fig. 10).

Toutefois, beaucoup de points d'analyse sont trop pauvres en matière colorante pour pouvoir conclure. Des mesures complémentaires et d'autres traitements plus spécifiques des données permettraient d'obtenir des résultats plus fins. En particulier, une meilleure sensibilité des analyses élémentaires permettrait de se prononcer sur les figures moins riches en matière colorante, tandis que des mesures structurales pourraient confirmer nos hypothèses quant à la nature des oxydes de manganèse utilisés.

\subsubsection{Iraitement des données du rouge et du jaune}

Le cas de figure du traitement des données de FX pour les tracés rouges et jaune est plus complexe d'une part car la paroi sous-jacente contient des concentrations non-négligeables en fer et, d'autre part, car la paroi de la grotte de Font-de-Gaume est très hétérogène et ne permet pas une approximation de sa composition chimique moyenne. Afin d'accéder directement à ces compositions chimiques, nous avons décidé de tester une autre méthode d'évaluation basée sur des simulations de Monte Carlo. Pour réaliser ces simulations, le logiciel XMI-MSIM a été utilisé. Le système « paroi et pigment » y est défini comme la superposition de trois couches : en partant du spectromètre, il y a une couche d'air, une couche de pigments et une couche de paroi. Cette vision est simpliste mais permet une première approche quantitative (GAY 2015 ; GAY et al. 2016).

La première étape a été de définir la couche de substrat, c'est-à-dire la composition chimique la constituant et la concentration des éléments présents. Un premier calcul de ces concentrations a été réalisé avec le logiciel PyMca, puis les concentrations ont été intégrées dans le logiciel de simulation Monte Carlo et ajustées pour que le spectre simulé concorde avec celui acquis expérimentalement. Nous avons dans ce cas-là un matériau constitué de deux couches, air et paroi. Ayant constaté une grande hétérogénéité de la paroi, cette première étape a été effectuée en amont de chaque modélisation des compositions chimiques des matières colorantes.

Une fois la paroi chimiquement bien définie, la couche de matière colorante peut être ajoutée au système modélisé. Cette deuxième étape comprend une estimation grossière, dans PyMca, de la concentration de chacun des éléments chimiques constituant la matière colorante exprimée sous forme d'oxyde, en soustrayant la contribution de la paroi. Cette première estimation, peu exacte, constitue tout de même une première base aux simulations de la couche contenant la matière colorante (fig. 11).

Il nous a alors été possible de distinguer la couche colorée de la couche sous-jacente (la paroi), et de différencier les signatures chimiques provenant de chacune. Les concentrations des différents éléments présents au sein des matières colorantes analysées ont donc été extraites à partir des spectres expérimentaux (tabl. 4 et 5). Nous pouvons d'ores et déjà remarquer que les écart-types associés aux concentrations des matières colorantes rouges sont élevés. Cela est lié aux variations importantes de la teneur des éléments chimiques accompagnant le fer, observées précédemment sur les spectres de FX.

Les concentrations des différents éléments obtenues par simulation de Monte Carlo ont été ensuite traitées par analyse en composantes principales (ACP), afin de mettre en évidence des relations entre ces données selon certaines combinaisons de variables.

Dans le cas du pigment rouge, les deux principales composantes, permettant de regrouper le maximum de variance et donc le maximum d'information, sont faibles (fig. 12). La première composante principale (CP1) n'explique que $25,5 \%$ de la dispersion des données tandis que la seconde (CP2) n'en explique que 24,8 \%. L'hétérogénéité de la paroi et la faible présence de matière colorante analysée explique la dispersion des données et la grande incertitude de ces données observées. 

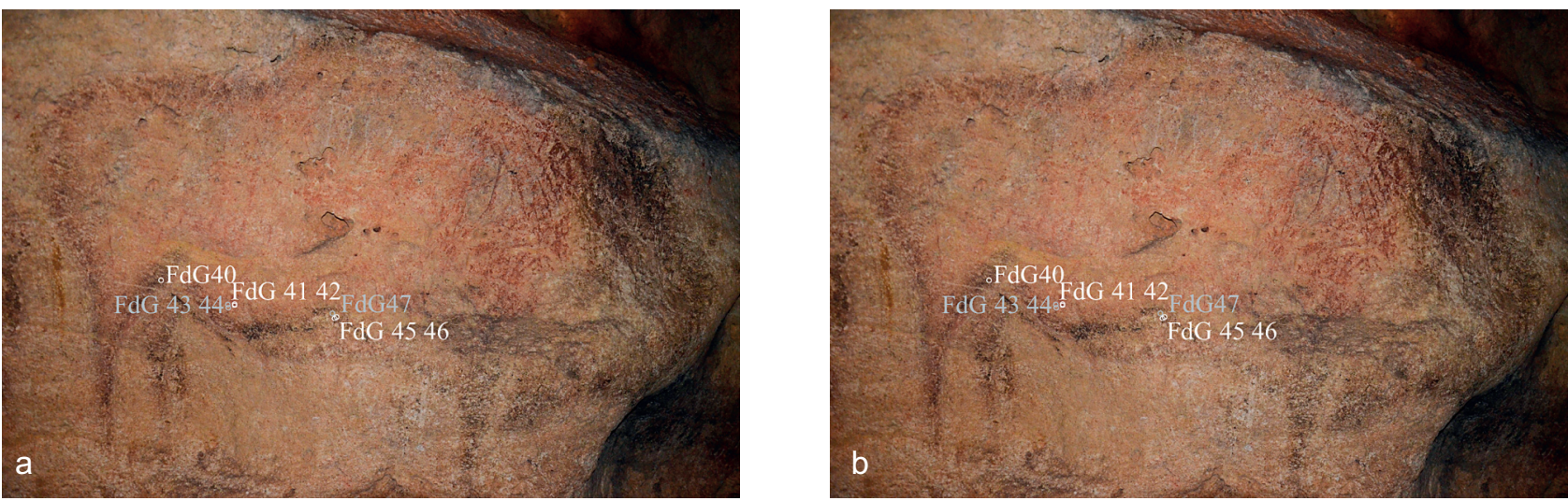

- FIGURE 7 -

Localisation des points d'analyse effectués sur la matière picturale et sur la paroi

Location of analysis points made on the pictorial material and on the wall on a) sur a) le bison 38 et b) le bison 39 de la grotte de Font-de-Gaume, ㄷ M. Gay, LAMS.

the bison 38 and b) the bison 39 of the cave of Font-de-Gaume, (c) M. Gay, LAMS.

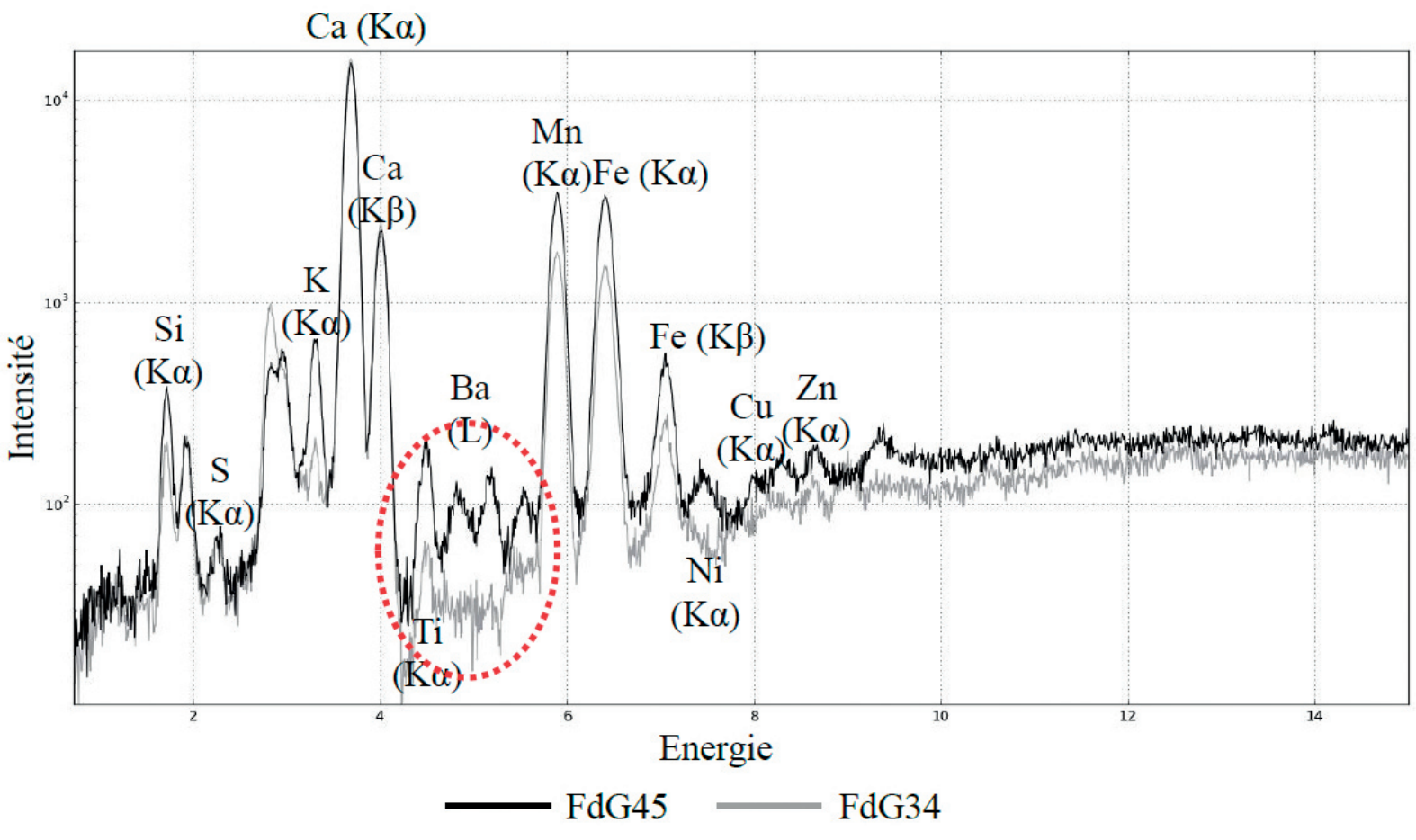

- FIGURE 8 -

Spectres de FX obtenus sur les tracés noirs des deux bisons 38 (FdG45) et 39 (FdG34) (cf. Figure 7 pour leur localisation précise), @ M. Gay, LAMS

Ainsi, il est difficile à ce stade de discriminer chimiquement les tracés rouges des figures analysées. Ceci peut être lié au faible nombre de figures rouges analysées dans la grotte pour le moment. De plus, il est possible que nous ayons analysé en premier, du fait de la facilité de leur accès, les figures rouges du même type et du même style car elles se trouvent principalement dans la galerie principale de la grotte, ce qui expliquerait pourquoi un seul type de matière colorante a été trouvé. Nous allons par la suite multiplier les analyses chimiques d'autres figures plus en hauteur et à différents endroits de la grotte
$X$-ray fluorescence spectra obtained on the black lines of two different polychrome bisons, the bisons 38 (FdG45) and 39 (FdG34) (see Figure 7 for their precise location) (c) M. Gay, LAMS.

présentant d'autres épaisseurs de matière colorante et des teintes variables déjà visibles à l'œil nu afin de disposer d'un jeu de données plus conséquent.

Néanmoins, ces premières observations combinées à des analyses non-invasives nous indiquent un potentiel de discrimination des figures préhistoriques grâce à l'analyse de leur style, de leur technique d'application et leur composition chimique analysable par FX in situ. 


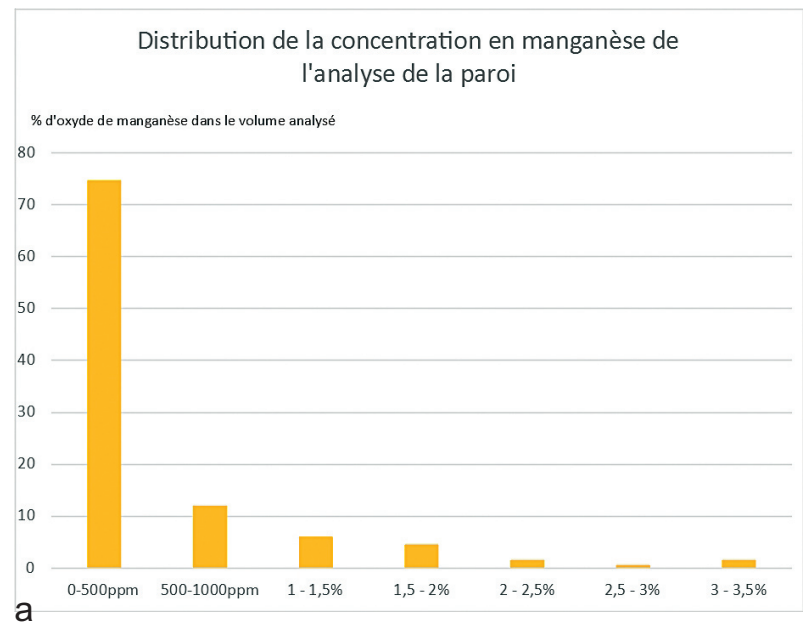

- FIGURE 9 -

Répartition de la concentration en manganèse des points d'analyses a) de la paroi et b) des zones colorées.

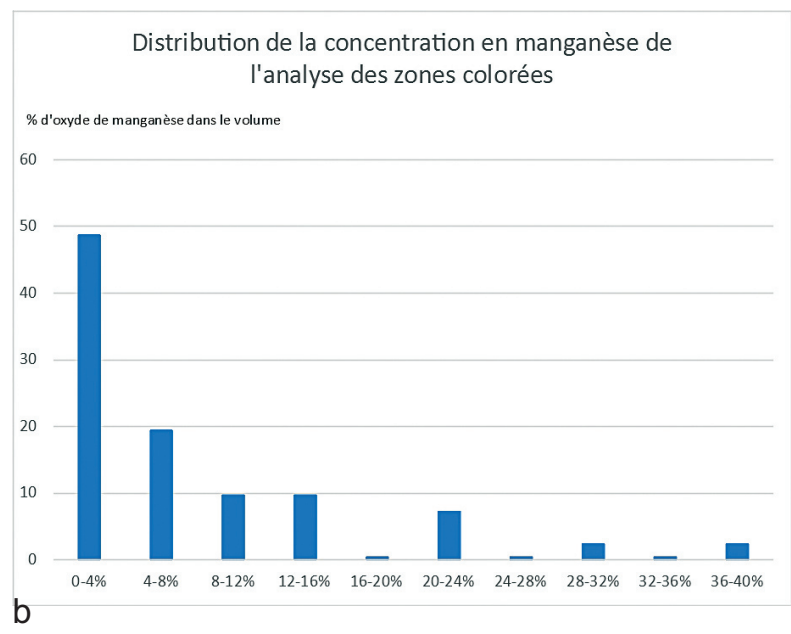

Distribution of the concentration of manganese in the analytical points a) in the wall and $b$ ) in the coloured traces.
Grotte de Font-de-Gaume

Répartition des figures

(d'après les bandes générales de Breuil in Capitan, Breuil et peyrony, 1910)

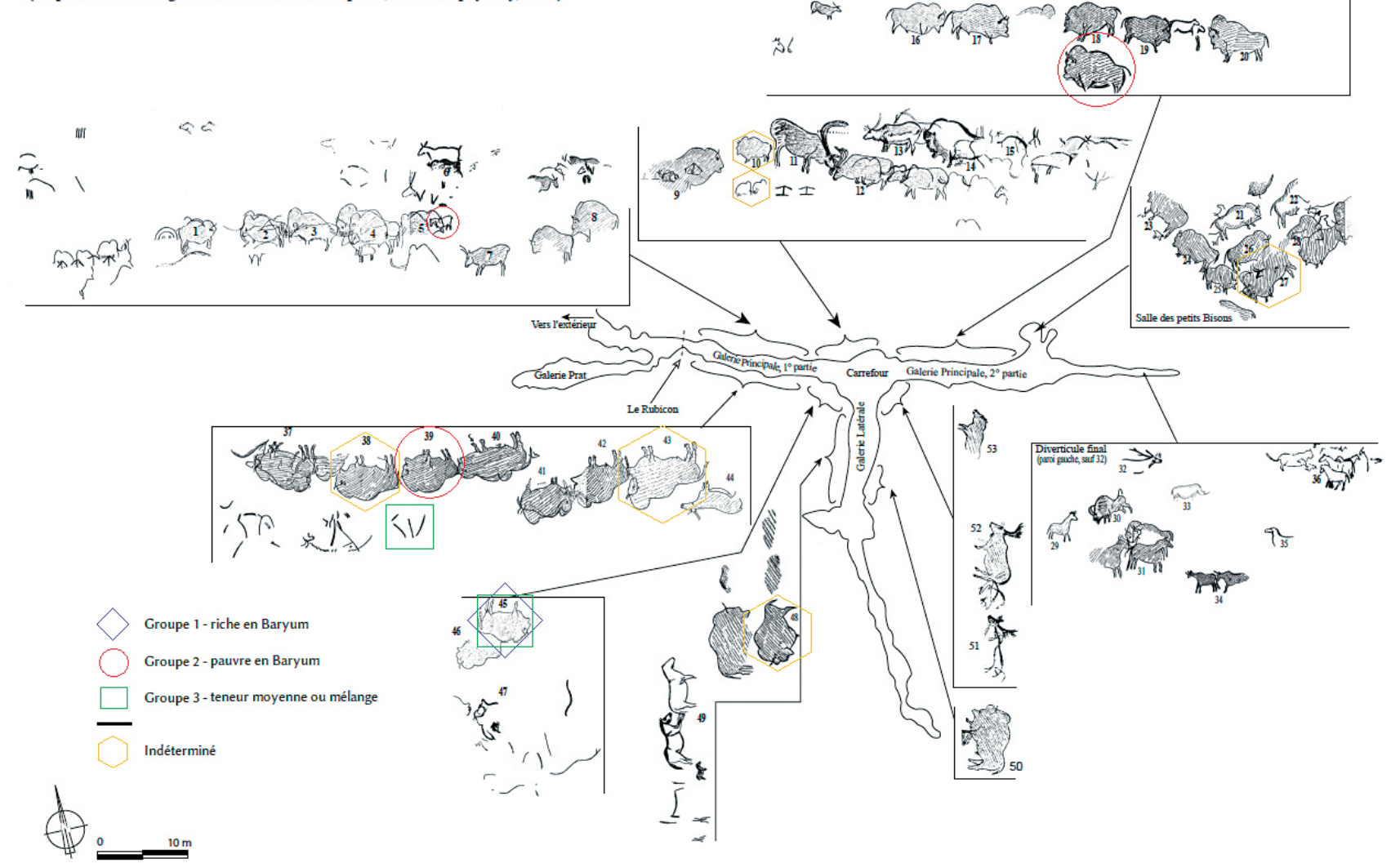

- FIGURE $10-$

Plan de répartition des figures analysées contenant des tracés noirs et regroupements selon la teneur en baryum des oxydes de manganèse utilisés.
Distribution of the figures analysed in the cave of Font-de-Gaume containing black traces and grouping as a function of the barium amount in the used manganese oxides, (c) A. Trosseau, IRCP / C2RMF. 

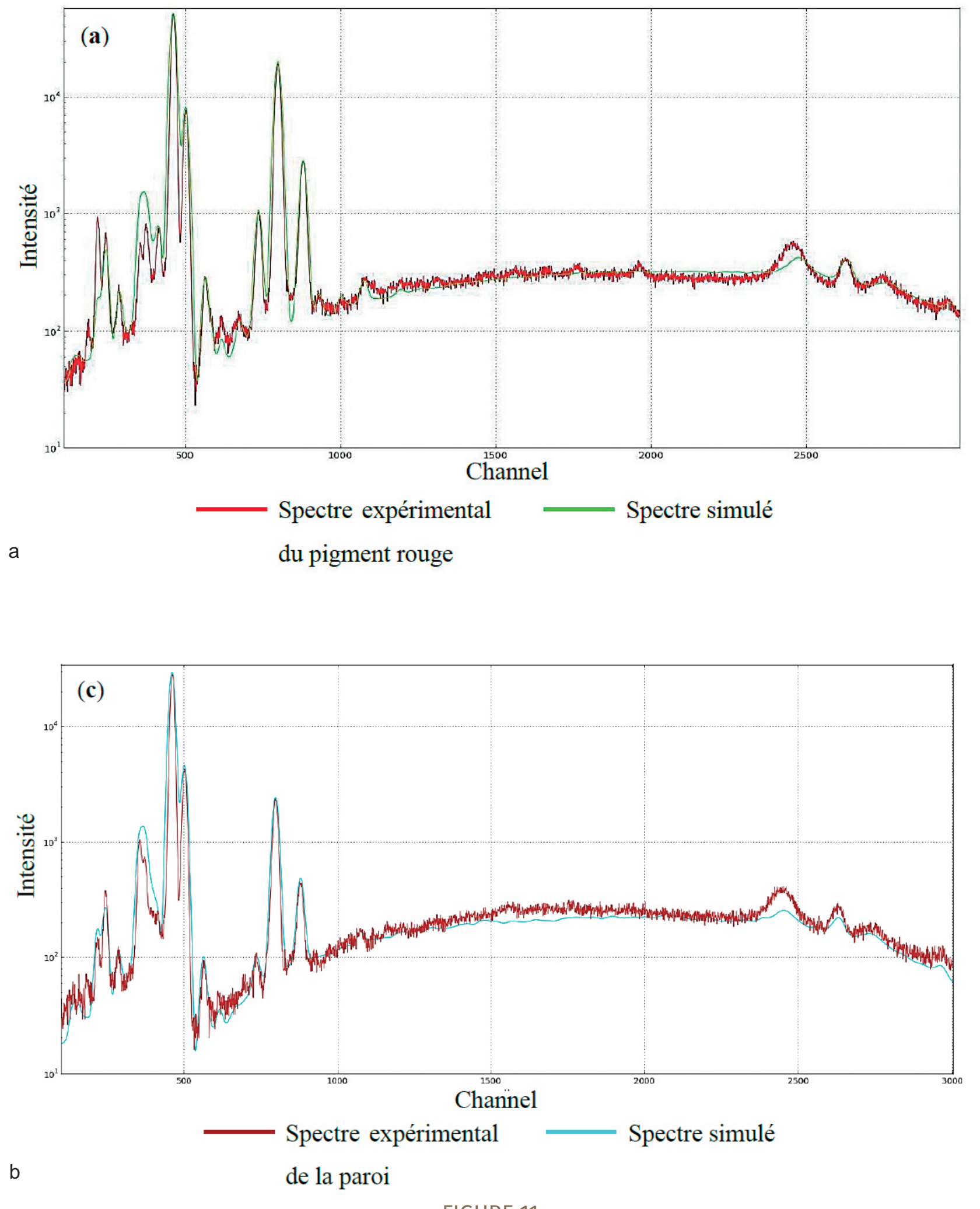


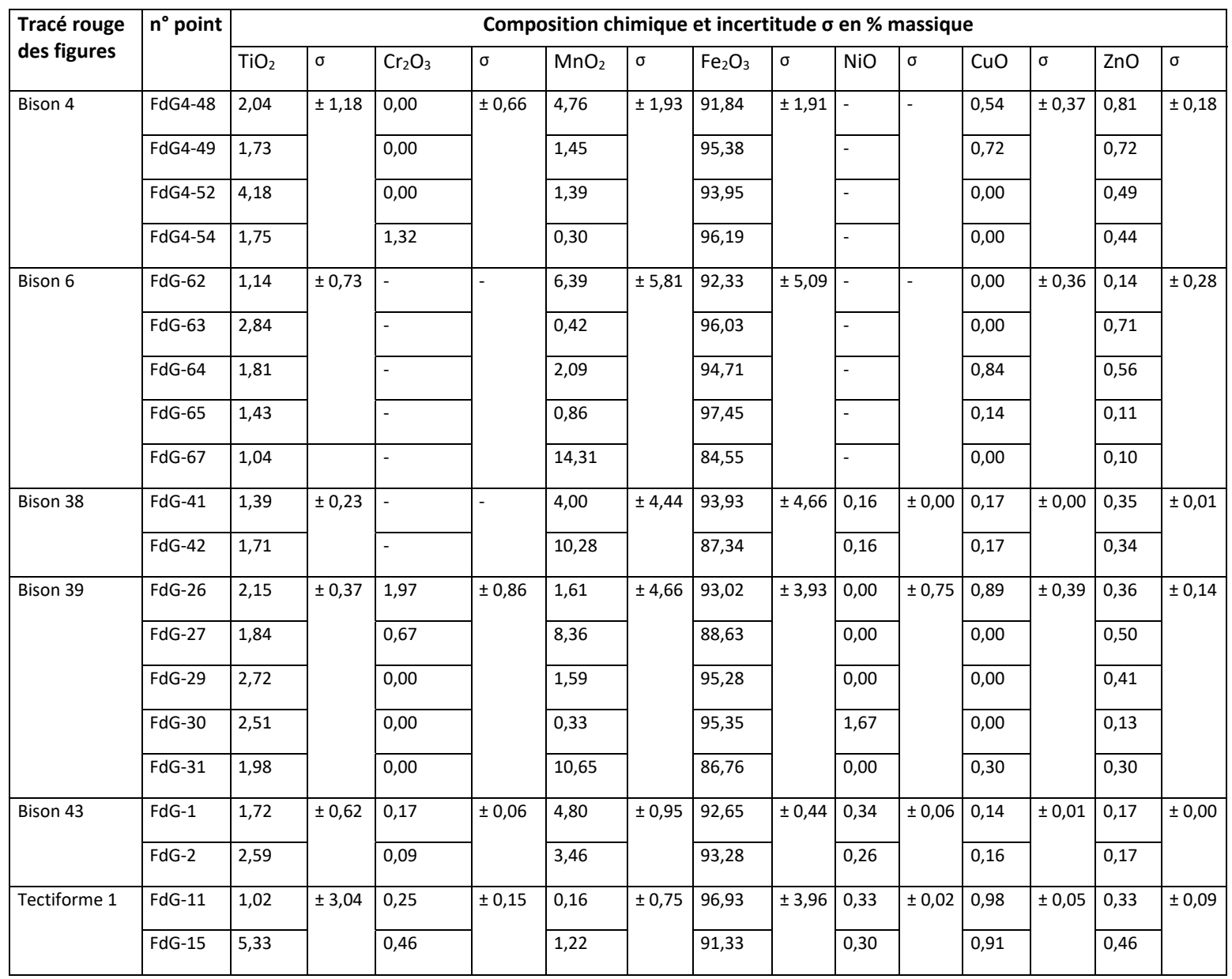

\section{- TABLEAU 4 -}

Analyse quantitative des données acquises par FX à l'aide du logiciel XMI-MSIM pour les tracés rouges des figures. La valeur de $\sigma$ est dans certain cas plus grande que la valeur de la concentration mesurée. Cela est dû à l'hétérogénéité de la composition de la paroi sous-jacente.
Quantitative analysis of the data acquired by PXRF using the XMI-MSIM software for the red traces of the figures. The value of $\sigma$ is in some cases larger than the value of the measured concentration. This is due to the heterogeneity of the composition of the underlying wall.

\begin{tabular}{|c|c|c|c|c|c|c|c|c|c|c|c|c|c|c|c|}
\hline \multirow{2}{*}{$\begin{array}{l}\text { Tracé noir } \\
\text { des figures }\end{array}$} & \multirow[t]{2}{*}{$n^{\circ}$ point } & \multicolumn{14}{|c|}{ Composition chimique et incertitude $\sigma$ en $\%$ massique } \\
\hline & & $\mathrm{TiO}_{2}$ & $\sigma$ & $\mathrm{MnO}_{2}$ & $\sigma$ & $\mathrm{Fe}_{2} \mathrm{O}_{3}$ & $\sigma$ & $\mathrm{NiO}$ & $\sigma$ & $\mathrm{CuO}$ & $\sigma$ & $\mathrm{ZnO}$ & $\sigma$ & $\mathrm{BaO}$ & $\sigma$ \\
\hline \multirow[t]{3}{*}{ Bison 43} & FdG-4 & 0,47 & \multirow[t]{3}{*}{ $\pm 0,27$} & 33,00 & \multirow[t]{3}{*}{ $\pm 10,54$} & 61,28 & \multirow[t]{3}{*}{ $\pm 12,21$} & 0,31 & \multirow[t]{3}{*}{ $\pm 0,17$} & 0,06 & \multirow[t]{3}{*}{ $\pm 0,02$} & 0,94 & \multirow[t]{3}{*}{ $\pm 0,24$} & 3,93 & \multirow[t]{3}{*}{ $\pm 1,54$} \\
\hline & FdG-5 & 0,93 & & 53,97 & & 37,01 & & 0,62 & & 0,08 & & 0,46 & & 6,94 & \\
\hline & FdG-6 & 0,45 & & 45,27 & & 46,78 & & 0,60 & & 0,10 & & 0,75 & & 6,03 & \\
\hline \multirow[t]{2}{*}{ Bison 38} & FdG-45 & 0,70 & \multirow[t]{2}{*}{ $\pm 0,12$} & 59,44 & \multirow[t]{2}{*}{ $\pm 1,54$} & 31,47 & \multirow[t]{2}{*}{ $\pm 1,09$} & 0,52 & \multirow[t]{2}{*}{ $\pm 0,00$} & 0,17 & \multirow[t]{2}{*}{ $\pm 0,12$} & 0,70 & \multirow[t]{2}{*}{ $\pm 0,13$} & 6,99 & \multirow[t]{2}{*}{ $\pm 0,59$} \\
\hline & FdG-46 & 0,53 & & 61,62 & & 29,93 & & 0,53 & & 0,35 & & 0,88 & & 6,16 & \\
\hline \multirow[t]{4}{*}{ Bison 39} & FdG-33 & 1,74 & \multirow[t]{4}{*}{ $\pm 0,35$} & 70,91 & \multirow[t]{4}{*}{ $\pm 7,02$} & 26,05 & \multirow[t]{4}{*}{ $\pm 5,69$} & - & \multirow[t]{4}{*}{-} & 0,72 & \multirow[t]{4}{*}{ $\pm 1,28$} & 0,58 & \multirow[t]{4}{*}{ $\pm 0,08$} & - & \multirow[t]{4}{*}{-} \\
\hline & FdG-34 & 1,61 & & 71,64 & & 26,32 & & - & & 0,00 & & 0,44 & & - & \\
\hline & FdG-36 & 1,42 & & 61,08 & & 34,09 & & - & & 2,84 & & 0,57 & & - & \\
\hline & FdG-37 & 0,95 & & 78,09 & & 20,23 & & - & & 0,31 & & 0,42 & & - & \\
\hline
\end{tabular}

\section{- TABLEAU 5 -}

Analyse quantitative des données acquises par FX à l'aide du logiciel XMI-MSIM Quantitative analysis of the data acquired by pXRF using the XMI-MSIM software pour les tracés noirs des figures. 

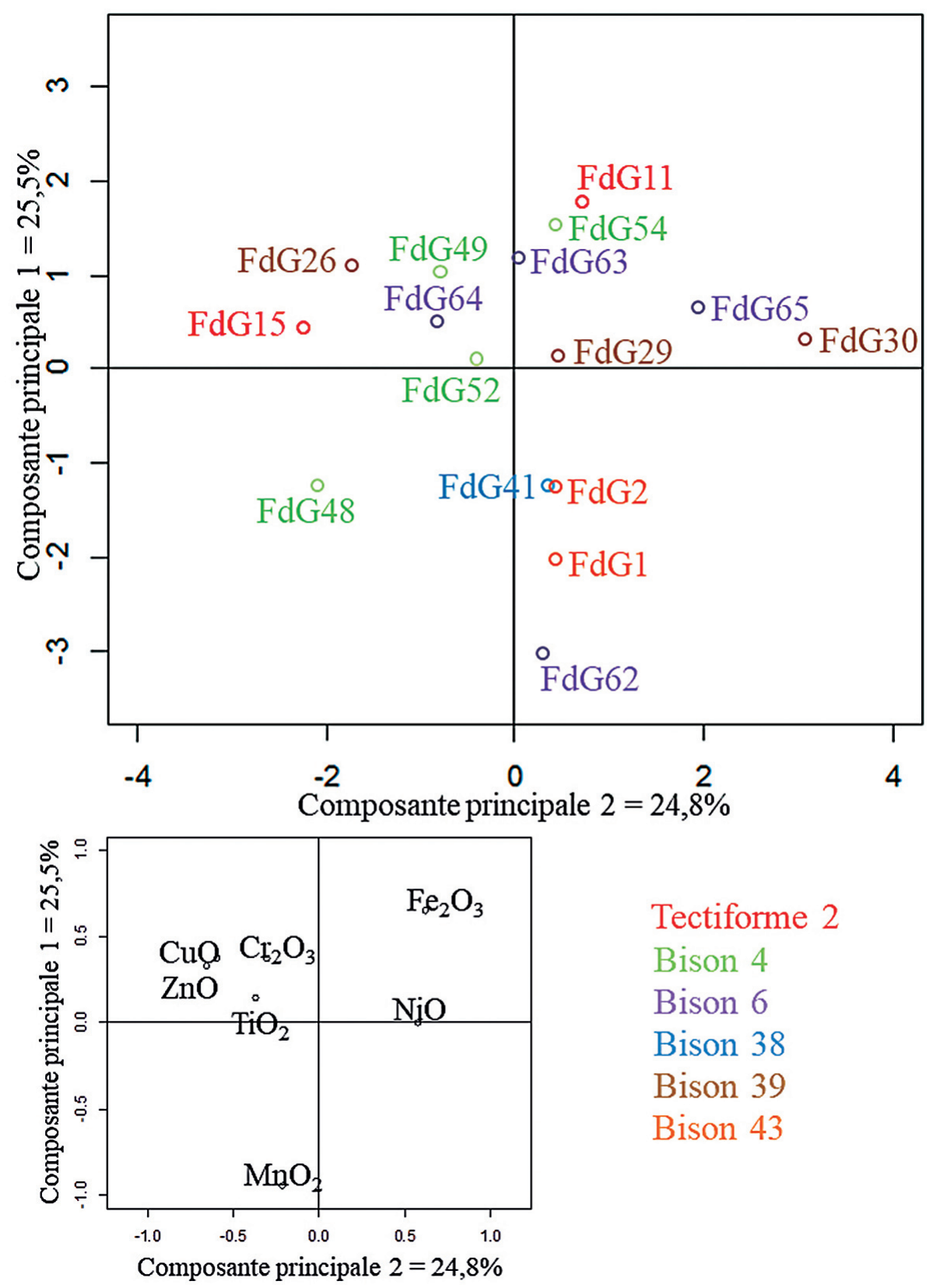

\section{Tectiforme 2}

Bison 4

Bison 6

Bison 38

Bison 39

Bison 43

\section{5 | CONCLUSIONS ET PERSPECTIVES}

Les premiers résultats d'analyse du décor de la grotte de Font-de-Gaume sont encourageants afin d'obtenir davantage d'informations sur la connaissance et les techniques de ces œuvres du Paléolithique supérieur. Pour les figures rouges, la dispersion importante de leur composition chimique déterminée, qui s'explique par la forte hétérogénéité de la paroi mise en évidence, empêche pour le moment la distinction chimique claire de figures analysées. Nous sommes amenés à penser que de fortes différences de composition chimique entre matières colorantes rouges auraient conduit à une distinction de groupes au sein des représentations étudiées. Ces matières colorantes étudiées, de la première partie de la galerie principale, semblent donc, de par leur composition chimique détectée en FX portable, relativement homogènes. D'autres approches de traitement des données semi-quantitatif sont nécessaires afin d'affiner ces résultats. Nos observations à l'œil nu des tracés rouges de différentes figures présentant des épaisseurs et des teintes variables dans les différentes parties de la grotte nous indiqueraient cependant qu'une utilisation de différents « pots de peintures » et donc une discrimination chimique de la matière colorante des tracés rouges devrait être possible. Ainsi, des analyses d'un plus grand nombre de figures présentant différentes techniques d'application et de styles sont à envisager, combinées avec une optimisation des traitements de données afin de pouvoir mieux distinguer différentes figures rouges et jaunes dans les différentes parties de la grotte.

Quant aux figures noires, les résultats ont été plus concluants. Trois types de matières colorantes ont pu être distingués présentant principalement différentes teneurs en baryum associé au manganèse. Ce résultat est cohérent avec ceux obtenus sur les matières colorantes identifiées issues d'autres grottes de la région datant du Magdalénien, notamment la grotte de Lascaux et la grotte de Rouffignac. 
Une approche holistique combinant observation détaillée de la paroi et des techniques d'application des figures préhistoriques, analyses de styles et analyses chimiques in situ permettra à terme, une fois l'ensemble du décor de la grotte étudié d'une façon systématique, de proposer un groupement de différentes figures ainsi qu'une séquence de leur réalisation. Ceci permettrait d'améliorer la connaissance de ces œuvres et de proposer éventuellement une datation relative dans le cas de superpositions des figures, ce qui paraît très important en l'absence d'une datation directe des œuvres pariétales de Font-de-Gaume.

D'autres analyses complémentaires et un traitement plus fin des données déjà acquises sont en cours de réalisation dans le cadre de la thèse d'Antoine Trosseau, débutée à la fin 2018. Nous espérons que les résultats pourront alors être améliorés en optimisant le traitement informatique mais surtout directement lors de l'acquisition des données, en faisant varier les conditions expérimentales grâce à l'utilisation de spectromètres de terrain de plus en plus performants. L'utilisation d'autres techniques d'analyse non-invasives lors de missions de terrain futures en complément de la spectrométrie de fluorescence $X$ (spectrométries Raman, diffraction des rayons $X$...) est également une piste à explorer, car elles peuvent apporter d'autres types d'informations.

\section{REMERCIEMENTS}

Nous remercions chaleureusement les guides de la grotte et Georges Levy de nous avoir soutenus activement lors des missions, le DIM Analytics pour le soutien financier pour la thèse de Marine Gay (2012/2015, ED388 UPMC) et PSL pour le soutien financier de la thèse d'Antoine Trosseau (2018, ED388, PSL).

\section{RÉFÉRENCES BIBLIOGRAPHIQUES}

BECK L., ROUSSELIĖRE H., CASTAING J., DURAN A., LEBON M., LAHLIL S., PLASSARD F. 2012 - Analyse in situ des dessins préhistoriques de la grotte de Rouffignac par fluorescence $X$ et diffraction X portable. ArcheoSciences. Revue d'archéométrie, 36, p. 139-152, fig., tabl., pl.

BECK L., ROUSSELIÈRE H., CASTAING J., DURAN A., LEBON M., MOIGNARD B., PLASSARD F. 2014 - First Use of Portable System Coupling X-Ray Diffraction and X-Ray Fluorescence for in-Situ Analysis of Prehistoric Rock Art. Talanta, 129, p. 459-464, fig., tabl., pl.

CAPITAN L., BREUIL H., PEYRONY D. 1910 - La Caverne de Font-de-Gaume Aux Eyzies Dordogne. Monaco : Chêne, 452 p., ill.

CLEYET-MERLE J.-J. 2014 - La grotte de Font-de-Gaume. Paris : Ed. du Patrimoine - Centre des Monuments Nationaux, $64 \mathrm{p}$.

CLOTTES J. 2008 - L'art des cavernes préhistoriques; Beauxarts - thèmes; PHAIDON, 2008.
DE SANOIT J., CHAMBELLAN D., PLASSARD F. 2005 Caractérisation in situ du pigment noir de quelques œuvres pariétales de la Grotte de Rouffignac à l'aide d'un système portable d'analyse par fluorescence X (XRF). Archeosciences. Revue d'archéométrie 2005, 29, 61-68.

CHALMIN E., MENU M.,VIGNAUD C. 2003 - Analysis of rock art painting and technology of Palaeolithic painters. Measurement Science and Technology, 2003, Nº

GAY M. 2015 - Développement de nouvelles procédures quantitatives pour une meilleure compréhension des pigments et des parois des grottes ornées préhistoriques, thèse Université Pierre et Marie Curie, ED388, 2015.

GAY M., MÜLLER K., PLASSARD F., REICHE I. 2016 - Efficient quantification procedures for data evaluation of portable X-ray fluorescence - Potential improvements for Palaeolithic cave art knowledge. J Archaeol Sci: Reports. 10, 878-886.

GAY M., PLASSARD F., MÜLLER K., REICHE I. 2020 - Relative chronology of Palaeolithic drawings of the Great Ceiling, Rouffignac cave, by chemical, stylistic and superimposition studies, J Archaeol Sci: Reports.102006.

KONIK S., LAFON-PHAM D., RISS I., AUJOULAT N., FERRIER C., KERVAZO B., PLASSARD F., REICHE I. 2014 - Etude des vermiculations par caractérisation morphologique, chromatique et chimique. L'exemple des grottes de Rouffignac et de Font-de-Gaume (Dordogne, France). Paleo Revue d'Archéologie Préhistorique 2014, nº spécial, "Microanalyses et datations de l'art préhistorique dans son contexte archéologique".

MAURAN G., LEBON M., DETROIT F., CARON B., NANKELA A., PLEURDEAU D., BAHAIN J.-J. 2019 - First in situ pXRF analyses of rock paintings in Erongo, Namibia: results, current limits, and prospects. Archaeological and Anthropological Sciences, 2019, No.11, 4123-4145.

CHANTERAUD C., CHALMIN E., HOERLE S., SALOMON H., MONNEY J. 2019 - Première étude comparative des matières colorantes issues des fouilles et des parois ornées de la Grotte aux Points. Karstologia, 2019, nº 73.

HUNTLEY J., AUBERT M., ROSS J., BRAND H.E.A., MORWOOD M.J. 2015 - One Colour, (at Least) Two Minerals: A Study of Mulberry Rock Art Pigment and a Mulberry Pigment 'Quarry' from the Kimberley, Northern Australia. Archaeometry, 57(1), 77-99

LAHLIL S., LEBON M., BECK L., ROUSSELIĖRE H., VIGNAUD C., REICHE I., MENU M., PAILLET P., PLASSARD F. 2012 - The first in situ micro-Raman spectroscopic analysis of prehistoric cave art of Rouffignac St-Cernin, France. Journal of Raman Spectroscopy, 43 (11), 1637-1643.

LEROI-GOURHAN A., VERTUT J. 1965 - Préhistoire de l'art occidental ; L'art et les grandes civilisations; L. Mazenod: Paris.

MOISSAN H. 1902 - Sur les matières colorantes des figures de la Grotte de Font-de-Gaume. Compte-rendu de l'académie des sciences, nº 134, 1539-1540. (13) 
PAILLET P. 1993 - Les traitements magdaléniens de l'image du Bison dans l'art pariétal et mobilier du Périgord. Nouvelle Approche d'un thème du bestiaire paléolithique. Thèse, Paris, Muséum national d'histoire naturelle.

PLASSARD F. 2005 - Les grottes ornées de Combarelles, Font-de-Gaume, Bernifal et Rouffignac: Contexte Archéologique, Thèmes et Style Des Représentations.

SALOMON H., COQUINOT Y., BECK L., VIGNAUD C., LEBON M., ODIN G., MATHIS F., FRANCOIS M. 2014 - Stratégies spécialisées d'acquisition de pigments rouges durant le Châtelperronien de la grotte du Renne à Arcy-sur-Cure (Yonne, France). Paleo, Revue d'Archéologie Préhistorique, numéro spécial, "Micro-analyses et datations de l'art préhistorique dans son contexte archéologique".
STRIVAY D., CLAR M., RAKKAA S., HOCQUET F.-P., DEFEYT C. 2016 - Development of a translation stage for in situ noninvasive analysis and high-resolution imaging. Applied Physics A, 122: 950.

VIALOU D., VILHENA-VIALOU A. V. 2014 - Font-de-Gaume : le site des tectiformes. Paleo, Revue d'archéologie préhistorique, numéro spécial 2014, p . 219-230. 